CRYSTALLOGRAPHIC COMMUNICATIONS

ISSN 2056-9890

Received 3 June 2016

Accepted 20 June 2016

Edited by A. J. Lough, University of Toronto, Canada

Keywords: crystal structure; benzene-1,2-diamine; zinc chloride; co-crystal salt.

CCDC references: $1486732 ; 1486731$; 1486730

Supporting information: this article has supporting information at journals.iucr.org/e

\section{Structural characterization of two benzene-1,2- diamine complexes of zinc chloride: a molecular compound and a co-crystal salt}

\author{
Patricia L. Zick and David K. Geiger*
}

Department of Chemistry, SUNY-College at Geneseo, Geneseo, NY 14454, USA. *Correspondence e-mail: geiger@geneseo.edu

The structures of two zinc complexes containing bidentate benzene-1,2-diamine ligands are reported. (Benzene-1,2-diamine- $\left.\kappa^{2} N, N^{\prime}\right)$ dichloroidozinc, $\left[\mathrm{ZnCl}_{2}\left(\mathrm{C}_{6} \mathrm{H}_{8} \mathrm{~N}_{2}\right)\right]$, (I), displays a distorted tetrahedral coordination sphere for the metal cation. The diamine ligand and the $\mathrm{Zn}$ atom reside on a crystallographic mirror plane. In the 1:1 co-crystal salt trans-diaquabis(4,5dimethylbenzene-1,2-diamine- $\left.\kappa^{2} N, N^{\prime}\right)$ zinc chloride-4,5-dimethylbenzene-1,2diamine (1/1), $\left[\mathrm{Zn}\left(\mathrm{C}_{8} \mathrm{H}_{12} \mathrm{~N}_{2}\right)_{2}\left(\mathrm{H}_{2} \mathrm{O}\right)_{2}\right] \mathrm{Cl}_{2} \cdot 2 \mathrm{C}_{8} \mathrm{H}_{12} \mathrm{~N}_{2}$, (II), the zinc(II) complex cation exhibits a tetragonally distorted octahedral coordination sphere. The $\mathrm{Zn}$ atom sits on a crystallographically imposed inversion center and the diamine ligands are tilted $30.63(6)^{\circ}$ with respect to the $\mathrm{ZnN}_{4}$ plane. Both complexes exhibit extensive hydrogen bonding. In (I), a stacked-sheet extended structure parallel to (101) is observed. In (II), the co-crystallized diamine is hydrogenbonded to the complex cation via $\mathrm{O}-\mathrm{H} \cdots \mathrm{N}$ and $\mathrm{N}-\mathrm{H} \cdots \mathrm{N}$ linkages. These units are in turn linked into planes along $(200)$ by $\mathrm{O}-\mathrm{H} \cdots \mathrm{Cl}$ and $\mathrm{N}-\mathrm{H} \cdots \mathrm{Cl}$ hydrogen bonds.

\section{Chemical context}

Zinc complexes bearing aryl diimine and/or heterocyclic ligands have been shown to emit brightly in the blue region of the spectrum (DeStefano \& Geiger, 2016; Tan et al., 2012; Liu et al., 2010; Xu et al., 2008; Yue et al., 2006; Singh et al., 2011; Wang et al., 2010). These complexes have potential use in photooptical devices because of their high thermal stability and the ability to tune their color by varying ancillary ligands and coordination geometry (Xu et al., 2008). Most of the compounds explored have acetate ligands. Substituting acetate with halide ligands provides an avenue for modulating the electronic structure of the complex and, hence, the carrier transport character. Toward that end, we have characterized several zinc complexes possessing benzene-1,2-diamine ligands (Geiger, 2012; Geiger \& Parsons, 2014) and substituted benzimidazole ligands (DeStefano \& Geiger, 2016). The benzene-1,2-diamine-containing complexes previously reported have a monodentate diamine coordination mode. We report herein two new zinc complexes containing bidentate benzene-1,2-diamine ligands: (benzene-1,2-diamine- $\kappa^{2} N, N^{\prime}$ )dichloridozinc, (I), and the 1:1 co-crystal salt trans-diaquabis(4,5-dimethylbenzene-1,2-diamine- $\left.\kappa^{2} N, N^{\prime}\right)$ zinc chloride 4,5-dimethylbenzene-1,2-diamine, (II). 
<smiles>Cl[C]1Nc2ccccc2N1</smiles>

(I)

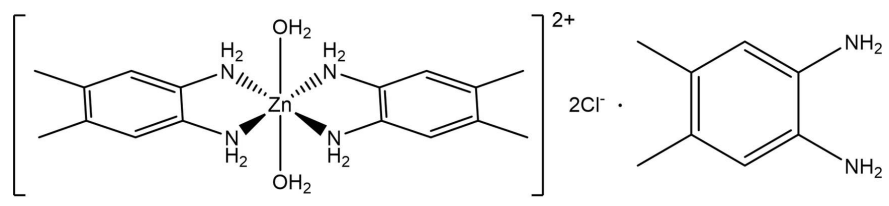

(II)

\section{Structural commentary}

As seen in Fig. 1, compound (I) exhibits a distorted tetrahedral coordination sphere for the metal cation. Tables 1 and 2 give relevant geometric parameters found in the coordination sphere. The diamine ligand and the $\mathrm{Zn}$ atom sit on a mirror plane and, hence, are rigorously planar as a result of the symmetry constraint. The $\mathrm{Zn}-\mathrm{N}$ bond lengths observed at the two temperatures are the same within the calculated s.u.s. The $\mathrm{Zn}-\mathrm{Cl}$ bond lengths differ within the s.u.s, with the $200 \mathrm{~K}$ structure being $0.0030(5) \AA$ longer. The bond lengths observed at both temperatures fall within the s.u. of the average value [2.221 (19) $\AA$ ] of similar complexes but the $\mathrm{Cl}-$ $\mathrm{Zn}-\mathrm{Cl}$ bond angles are smaller than the average of the values $\left[115(1)^{\circ}\right]$ reported for similar $\mathrm{Zn}^{\mathrm{II}}$ dichlorides in a tetrahedral environment (Shi et al., 2010; You, 2005; Lee et al., 2007).

Compound (II) consists of a $\mathrm{Zn}^{\mathrm{II}}$ complex cation with two bidentate 4,5-dimethylbenzene-1,2-diamine ligands and trans water ligands, chloride counter-ions and a non-coordinating molecule of 4,5-dimethylbenzene-1,2-diamine. The compound is thus classified as a co-crystal salt (Grothe et al., 2016). A representation of (II) is found in Fig. 2. The $\mathrm{Zn}^{\mathrm{II}}$ ion sits on a crystallographically imposed center of symmetry and has a

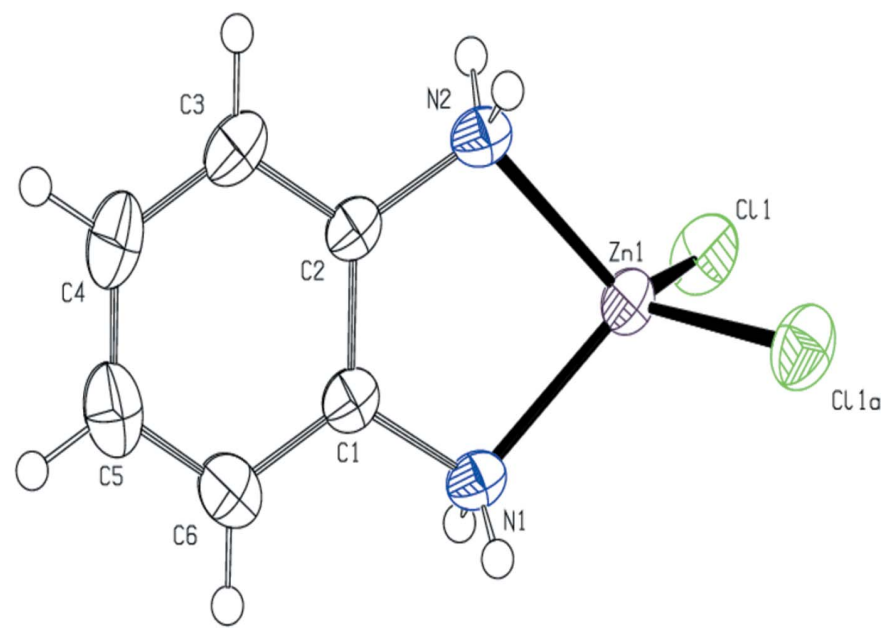

Figure 1

The molecular structure of ( $\mathrm{I} a$ ), showing the atom-labeling scheme. Anisotropic displacement parameters are drawn at the $50 \%$ probability level. [Symmetry code: $(a) x,-y+\frac{3}{2}, z$.]
Table 1

Selected geometric parameters $\left(\AA{ }^{\circ}\right)$ for $(\mathrm{I} a)$.

\begin{tabular}{lrll}
\hline $\mathrm{Zn} 1-\mathrm{Cl} 1$ & $2.2271(5)$ & $\mathrm{Zn} 1-\mathrm{N} 2$ & $2.0454(18)$ \\
$\mathrm{Zn} 1-\mathrm{N} 1$ & $2.0449(19)$ & & \\
& & & \\
$\mathrm{Cl} 1-\mathrm{Zn} 1-\mathrm{Cl} 1^{\mathrm{i}}$ & $110.82(2)$ & $\mathrm{N} 1-\mathrm{Zn} 1-\mathrm{Cl} 1$ & $113.82(3)$ \\
$\mathrm{N} 1-\mathrm{Zn} 1-\mathrm{N} 2$ & $85.53(8)$ & $\mathrm{N} 2-\mathrm{Zn} 1-\mathrm{Cl} 1$ & $115.42(3)$ \\
\hline
\end{tabular}

Symmetry code: (i) $x,-y+\frac{3}{2}, z$.

Table 2

Selected geometric parameters $\left(\AA{ }^{\circ}\right)$ for $(\mathrm{I} b)$.

\begin{tabular}{llll}
\hline $\mathrm{Zn} 1-\mathrm{Cl} 1$ & $2.2301(5)$ & $\mathrm{Zn} 1-\mathrm{N} 2$ & $2.045(3)$ \\
$\mathrm{Zn} 1-\mathrm{N} 1$ & $2.047(2)$ & &
\end{tabular}

$\mathrm{Cl} 1-\mathrm{Zn} 1-\mathrm{Cl}^{\mathrm{i}} \quad 110.70(3) \quad \mathrm{N} 1-\mathrm{Zn} 1-\mathrm{Cl} 1 \quad 113.89(4)$

$\mathrm{N} 1-\mathrm{Zn} 1-\mathrm{N} 2 \quad 85.45(10) \quad \mathrm{N} 2-\mathrm{Zn} 1-\mathrm{Cl} 115.46(3)$

Symmetry code: (i) $x,-y+\frac{3}{2}, z$.

Table 3

Selected geometric parameters $\left(\AA{ }^{\circ},{ }^{\circ}\right)$ for (II).

\begin{tabular}{lrll}
\hline $\mathrm{Zn} 1-\mathrm{N} 1$ & $2.1214(15)$ & $\mathrm{Zn} 1-\mathrm{O} 1$ & $2.2410(15)$ \\
$\mathrm{Zn} 1-\mathrm{N} 2$ & $2.1442(17)$ & & \\
$\mathrm{N} 1{ }^{\mathrm{i}}-\mathrm{Zn} 1-\mathrm{N} 2$ & $100.31(6)$ & $\mathrm{N} 1-\mathrm{Zn} 1-\mathrm{O} 1$ & $92.18(7)$ \\
$\mathrm{N} 1-\mathrm{Zn} 1-\mathrm{N} 2$ & $79.69(6)$ & $\mathrm{N} 2-\mathrm{Zn} 1-\mathrm{O} 1$ & $93.22(7)$ \\
\hline
\end{tabular}

Symmetry code: (i) $-x+1,-y+1,-z+1$.

tetragonally distorted octahedral coordination geometry. The observed $\mathrm{Zn}-\mathrm{O}$ bond length (Table 3 ) is significantly longer than the average of the values [2.14 (3) §] reported for similar trans aqua zinc(II) complexes (Necefoglu et al., 2001; İbrahim et al., 2006; Karimnejad et al., 2011; Gallardo et al., 2008; Li et $a l ., 2012$ ) and the range [2.008 (3) to 2.147 (3) $\AA$ ] found in the hexaaquazinc(II) cation (Lian et al., 2009). However, it is close to the 2.2057 (16) $\AA$ found in the similar cation of trans-diaquabis(cyclohexane-1,2-diamine)zinc dichloride (Karimnejad et al., 2011). The plane of the 4,5-dimethylbenzene-1,2-

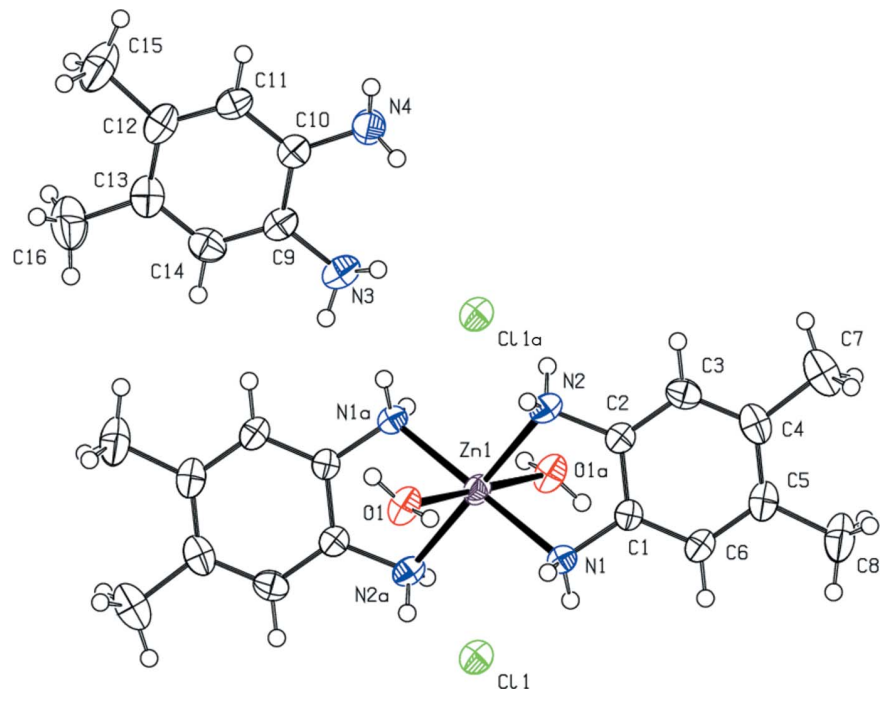

Figure 2

The molecular structure of (II) showing the atom-labeling scheme. Anisotropic displacement parameters are drawn at the $50 \%$ probability level. [Symmetry code: $(a)-x+1,-y+1,-z+1$.] 
Table 4

Hydrogen-bond geometry $\left(\AA,^{\circ}\right)$ for $(\mathrm{I} a)$.

\begin{tabular}{lllll}
\hline$D-\mathrm{H} \cdots A$ & $D-\mathrm{H}$ & $\mathrm{H} \cdots A$ & $D \cdots A$ & $D-\mathrm{H} \cdots A$ \\
\hline $\mathrm{N} 1-\mathrm{H} 1 \cdots \mathrm{Cl} 1^{\mathrm{ii}}$ & $0.86(2)$ & $2.59(2)$ & $3.3618(16)$ & $150.7(18)$ \\
$\mathrm{N} 2-\mathrm{H} 2 \cdots \mathrm{Cl} 1^{\mathrm{iii}}$ & $0.85(2)$ & $2.52(2)$ & $3.3204(16)$ & $157(2)$ \\
\hline
\end{tabular}

Symmetry codes: (ii) $x+\frac{1}{2}, y,-z+\frac{1}{2}$; (iii) $-x,-y+1,-z+1$.
Table 5

Hydrogen-bond geometry $\left(\AA,^{\circ}\right)$ for $(\mathrm{I} b)$.

\begin{tabular}{lllll}
\hline$D-\mathrm{H} \cdots A$ & $D-\mathrm{H}$ & $\mathrm{H} \cdots A$ & $D \cdots A$ & $D-\mathrm{H} \cdots A$ \\
\hline $\mathrm{N} 1-\mathrm{H} 1 \cdots \mathrm{Cl} 1^{\mathrm{ii}}$ & $0.83(3)$ & $2.61(3)$ & $3.368(2)$ & $152(2)$ \\
$\mathrm{N} 2-\mathrm{H} 2 \cdots \mathrm{C} 1^{\mathrm{iii}}$ & $0.83(3)$ & $2.53(3)$ & $3.327(2)$ & $160(2)$ \\
\hline
\end{tabular}

Symmetry codes: (ii) $x+\frac{1}{2}, y,-z+\frac{1}{2}$; (iii) $-x,-y+1,-z+1$.

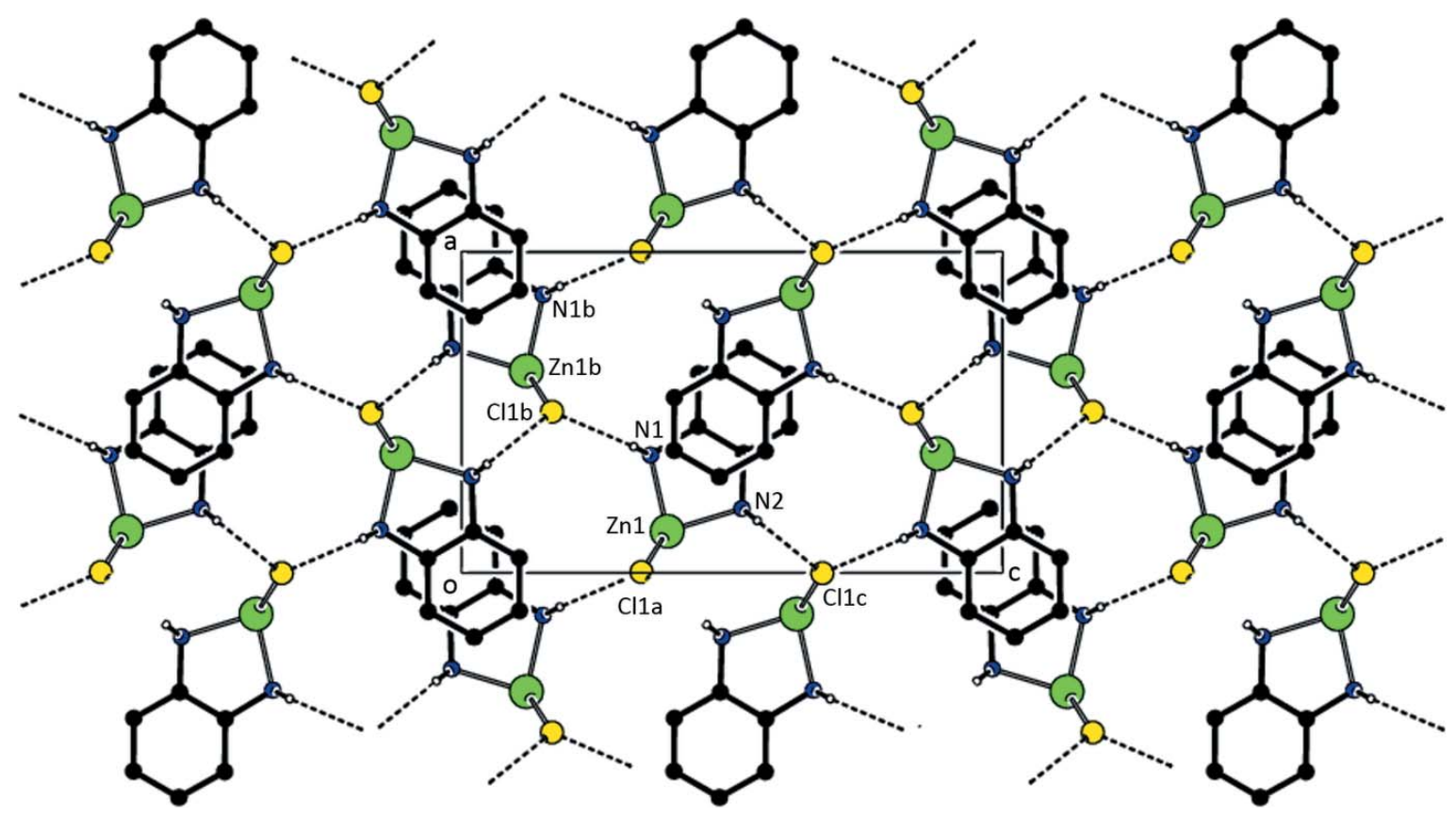

Figure 3

A view of the parallel sheets found in (I). Only $\mathrm{H}$ atoms involved in the $\mathrm{N}-\mathrm{H} \cdots \mathrm{Cl}$ interactions are shown. [Symmetry codes: $(a) x,-y+\frac{3}{2}, z ;(b) x+\frac{1}{2}$, $-y+\frac{3}{2},-z+\frac{1}{2} ;(c)-x,-y+1,-z+1$.]

Figure 4

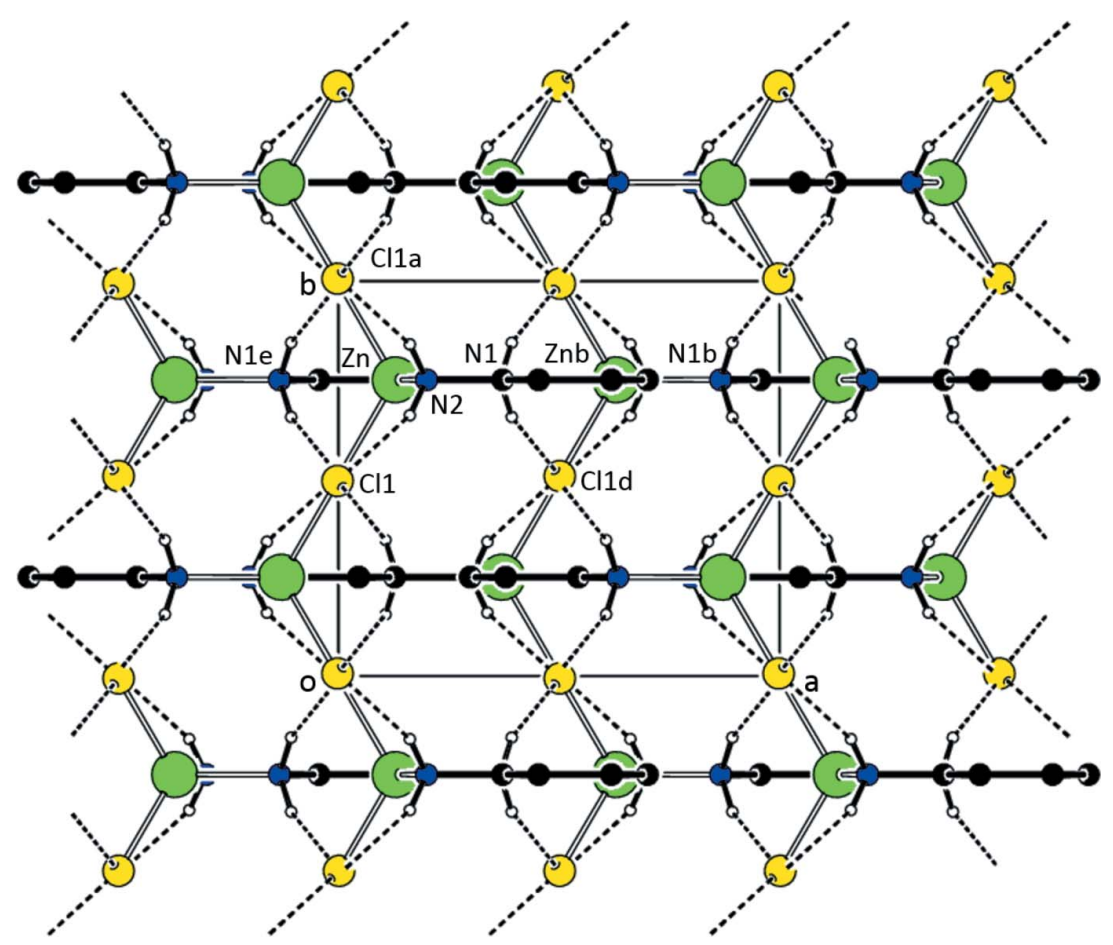

A view of the parallel sheets found in (I). Only $\mathrm{H}$ atoms involved in the $\mathrm{N}-\mathrm{H} \cdots \mathrm{Cl}$ interactions are shown. [Symmetry codes: $(a) x,-y+\frac{3}{2}, z ;(b) x+\frac{1}{2}$, $-y+\frac{3}{2},-z+\frac{1}{2} ;(d) x+\frac{1}{2}, y,-z+\frac{1}{2} ;(e) x-\frac{1}{2},-y+\frac{3}{2},-z+\frac{1}{2}$.] 
Table 6

Hydrogen-bond geometry $\left(\AA,^{\circ}\right)$ for (II).

\begin{tabular}{|c|c|c|c|c|}
\hline$D-\mathrm{H} \cdots A$ & $D-\mathrm{H}$ & $\mathrm{H} \cdots A$ & $D \cdots A$ & $D-\mathrm{H} \cdots A$ \\
\hline $\mathrm{O} 1-\mathrm{H} 1 W A \cdots \mathrm{N} 4^{\mathrm{ii}}$ & $0.81(3)$ & $2.13(3)$ & $2.924(3)$ & $164(2)$ \\
\hline $\mathrm{O} 1-\mathrm{H} 1 W B \cdots \mathrm{Cl} 1^{\mathrm{iii}}$ & $0.80(3)$ & $2.31(3)$ & $3.1083(17)$ & $173(2)$ \\
\hline $\mathrm{N} 1-\mathrm{H} 1 A \cdots \mathrm{Cl} 1^{\mathrm{iii}}$ & $0.84(2)$ & $2.55(2)$ & $3.3551(18)$ & $160.3(18)$ \\
\hline $\mathrm{N} 1-\mathrm{H} 1 B \cdots \mathrm{N} 3^{\text {iv }}$ & $0.85(2)$ & $2.31(2)$ & 3.137 (3) & $162.6(18)$ \\
\hline $\mathrm{N} 2-\mathrm{H} 2 A \cdots \mathrm{Cl} 1^{\mathrm{v}}$ & $0.87(2)$ & $2.63(2)$ & $3.4401(19)$ & $155.3(18)$ \\
\hline $\mathrm{N} 2-\mathrm{H} 2 B \cdots \mathrm{Cl} 1^{\mathrm{i}}$ & $0.81(2)$ & $2.57(2)$ & $3.3105(18)$ & $154(2)$ \\
\hline $\mathrm{N} 3-\mathrm{H} 3 A \cdots \mathrm{Cl1}^{\mathrm{v}}$ & $0.84(3)$ & $2.68(3)$ & $3.516(2)$ & $174(2)$ \\
\hline $\mathrm{N} 3-\mathrm{H} 3 B \cdots \mathrm{Cl} 1^{\mathrm{vi}}$ & $0.87(3)$ & $2.89(2)$ & $3.3284(19)$ & $112.9(18)$ \\
\hline $\mathrm{N} 4-\mathrm{H} 4 B \cdots \mathrm{Cl}^{\mathrm{v}}$ & $0.87(3)$ & $2.50(3)$ & $3.355(2)$ & $171(2)$ \\
\hline
\end{tabular}

Symmetry codes: (i) $-x+1,-y+1,-z+1$; (ii) $x,-y+\frac{3}{2}, z-\frac{1}{2}$; (iii) $x, y, z-1$; (iv) $-x+1, y-\frac{1}{2},-z+\frac{3}{2} ;(\mathrm{v})-x+1, y+\frac{1}{2},-z+\frac{3}{2}$; (vi) $-x+1,-y+1,-z+2$.

diamine ligand is canted $30.63(6) \AA$ out of the $\mathrm{ZnN}_{4}$ coordination plane. The nitrogen atoms of the diamine ligand are 0.022 (3) and 0.131 (3) $\AA$ out of the benzene plane for N1 and N2, respectively. For the co-crystallized diamine, N3 and N4 are 0.139 (3) and 0.088 (3) ^ out of the plane, respectively.

\section{Supramolecular features}

As seen in Figs. 3 and 4 and Tables 4 and 5, N1-H1 $\cdots \mathrm{Cl}$ hydrogen bonds between adjacent molecules result in strips of molecules of (I) along [100]. The strips form planes parallel to (101). Additional $\mathrm{N} 2-\mathrm{H} 2 \cdots \mathrm{Cl}$ bonds join the strips to form the three-dimensional network.

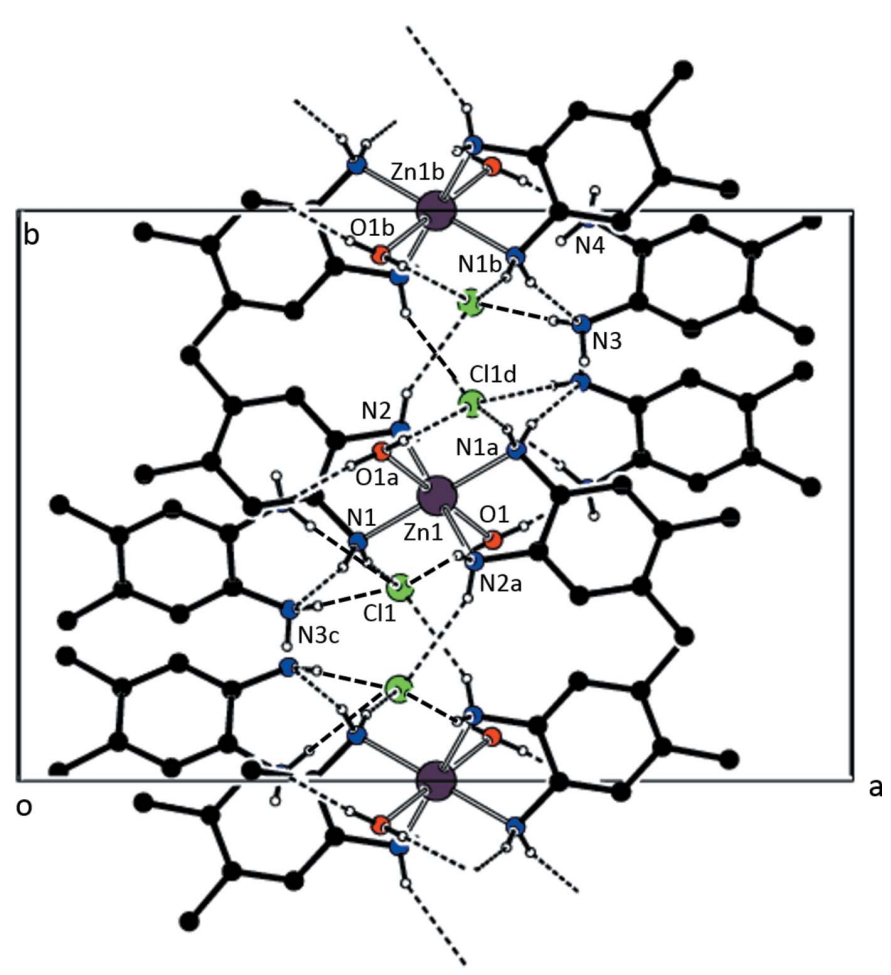

Figure 5

A view of the hydrogen-bonded network of (II) resulting in slabs along (200). Only $\mathrm{H}$ atoms bonded to the nitrogen atoms are shown. [Symmetry codes: $(a)-x+1,-y+1,-z+1 ;(b)-x+1, y+\frac{1}{2},-z+\frac{3}{2} ;(c)-x+1, y-\frac{1}{2}$, $-z+\frac{3}{2} ;(d)-x+1,-y+1,-z+2$.]
Fig. 5 presents a view of the hydrogen-bonding network in (II). $\mathrm{N}-\mathrm{H} \cdots \mathrm{N}$ and $\mathrm{O}-\mathrm{H} \cdots \mathrm{N}$ hydrogen bonds connect inversion-related co-crystallized 4,5-dimethylbenzene-1,2-diamine molecules to the complex cation (see Table 6). Additional $\mathrm{N}-\mathrm{H} \cdots \mathrm{Cl}$ and $\mathrm{O}-\mathrm{H} \cdots \mathrm{Cl}$ hydrogen bonds join the units, forming planes parallel to (200).

\section{Database survey}

The structures of the tetrahedral complexes bis(acetato$\kappa O)$ (benzene-1,2-diamine- $\kappa N$ )zinc (Mei et al., 2009) and bis(acetato- $\kappa O)(4,5$-dimethylbenzene-1,2-diamine- $\kappa N)$ zinc (Geiger, 2012) have been reported. Poly[[tris $\left(\mu_{2}\right.$-acetato$\left.\kappa^{2} O: O^{\prime}\right)(4$-chlorobenzene-1,2-diamine- $\kappa N)\left(\mu_{3}\right.$-hydroxido $)$ dizinc] ethanol monosolvate] exhibits alternating octahedral and tetrahedral zinc coordination modes (Geiger \& Parsons, 2014). Dichlorido[N-(2-pyridylmethylidene)benzene-1,4-diamine]zinc has a tetrahedral coordination sphere with intermolecular $\mathrm{N}-\mathrm{H} \cdots \mathrm{Cl}$ hydrogen bonds (Shi et al., 2010). Dichlorido[ $N, N, N^{\prime}, N^{\prime}$-tetramethylcyclohexane-1,2-diamine$\left.\kappa^{2} N, N^{\prime}\right]$ zinc displays a tetrahedral coordination geometry (Lee et al., 2007). For examples of zinc complexes with the metal in octahedral coordination including trans water ligands, see İbrahim et al. (2006); Necefoglu et al. (2001); Karimnejad et al. (2011). A tetragonally distorted octahedral zinc complex that contains both a mono- and a bidentate benzene-1,2-diamine ligand (Qian et al., 2007) and a distorted octahedral complex with trans monodentate benzene-1,2-diamine ligands (Ovalle-Marroquín et al., 2002) have been reported.

\section{Synthesis and crystallization}

Compound (I) was prepared by mixing a solution of $100 . \mathrm{mg}$ $(0.734 \mathrm{mmol})$ zinc chloride dissolved in approximately $5 \mathrm{~mL}$ ethanol with a solution of $238 \mathrm{mg}(2.20 \mathrm{mmol})$ benzene-1,2diamine dissolved in approximately $5 \mathrm{~mL}$ ethanol. The mixture became cloudy with a fine white precipitate. After the addition of 4 drops of $6 \mathrm{M} \mathrm{HCl}$, the mixture was gently heated, filtered and allowed to slowly evaporate. After two days, $0.0273 \mathrm{~g}$ ( $0.117 \mathrm{mmol}, 15 \%$ yield $)$ of clear, colorless crystals were isolated, which were used for data collection. The diffraction pattern showed signs of degradation as the temperature was lowered to $200 \mathrm{~K}$ from $300 \mathrm{~K}$ and so data sets were collected at both temperatures.

Compound (II) was prepared by combining solutions of $100 \mathrm{mg}$ (0.734 mmole) zinc chloride in a few $\mathrm{mL}$ of ethanol and $300 \mathrm{mg}$ (2.20 mmol) 4,5-dimethylbenzene-1,2-diamine in a few $\mathrm{mL}$ of ethanol. After the addition of 4 drops of $6 \mathrm{M} \mathrm{HCl}$, the mixture was gently heated and filtered. The filtrate was divided into three portions and each allowed to slowly evaporate. After several days, a small number of clear, colorless crystals in the shape of hexagonal plates were isolated, one of which was used for data collection. 
Table 7

Experimental details.

Crystal data

Chemical formula

$M_{\mathrm{r}}$

Crystal system, space group

Temperature (K)

$a, b, c(\AA)$

$\alpha, \beta, \gamma\left({ }^{\circ}\right)$

$V\left(\AA^{3}\right)$

$Z$

Radiation type

$\mu\left(\mathrm{mm}^{-1}\right)$

Crystal size (mm)

Data collection

Diffractometer

Absorption correction

$T_{\min }, T_{\max }$

No. of measured, independent and observed $[I>2 \sigma(I)]$ reflections

$R_{\text {int }}$

$(\sin \theta / \lambda)_{\max }\left(\AA^{-1}\right)$

0.039
0.658

Refinement

$R\left[F^{2}>2 \sigma\left(F^{2}\right)\right], w R\left(F^{2}\right), S$

No. of reflections

No. of parameters

$\mathrm{H}$-atom treatment

$\Delta \rho_{\max }, \Delta \rho_{\min }\left(\mathrm{e} \AA^{-3}\right)$
(Ia)

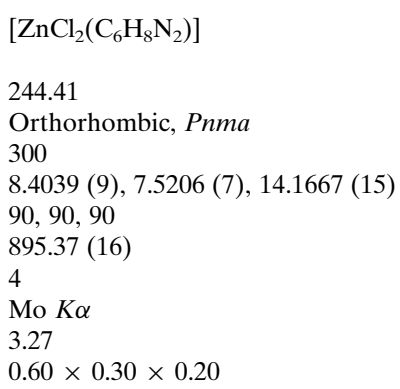

$(\mathrm{I} b)$

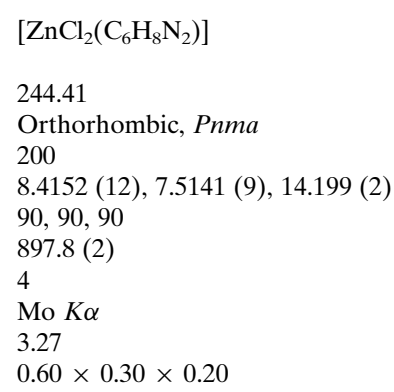

Bruker SMART X2S benchtop Multi-scan (SADABS; Bruker, 2013)

$0.39,0.56$

$9435,1129,1026$

$0.020,0.051,1.09$

1129

72

$\mathrm{H}$ atoms treated by a mixture of independent and constrained refinement

$0.26,-0.40$

Bruker SMART X2S benchtop Multi-scan (SADABS; Bruker, 2013)

$0.40,0.56$

4392, 1090, 992

0.040

0.649

$0.027,0.074,1.16$

1090

72

$\mathrm{H}$ atoms treated by a mixture of independent and constrained refinement

$0.41,-0.58$
(II)

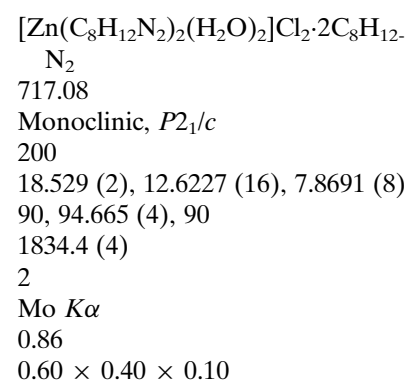

Bruker SMART X2S benchtop
Multi-scan (SADABS; Bruker,
2013)
$0.66,0.92$
$25430,3619,2920$
0.060
0.617

$0.031,0.079,1.05$
3619
245
$\mathrm{H}$ atoms treated by a mixture of
independent and constrained
refinement
$0.31,-0.22$

Bruker SMART X2S benchtop Multi-scan (SADABS; Bruker,

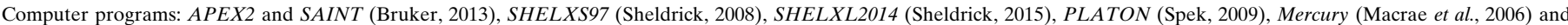
publCIF (Westrip, 2010).

\section{Refinement details}

Crystal data, data collection and structure refinement details are summarized in Table 7. For compound (I), data sets were collected at $300 \mathrm{~K}(\mathrm{I} a)$ and $200 \mathrm{~K}(\mathrm{I} b)$. The diffraction pattern showed clear degradation at the lower temperature. Examination of the crystal subjected to the cold stream showed fractures that were not previously present. As seen in Table 7, the cell constant s.u.s, $R$ values and $S$ values are lower for the $300 \mathrm{~K}$ data set.

For both (I) and (II), all hydrogen atoms were located in difference Fourier maps. For (I), all hydrogen atoms bonded to the nitrogen atoms were refined freely, including isotropic displacement parameters. For $(\mathrm{I} a)$, the hydrogen atoms bonded to the benzene carbon atoms were refined using a riding model with $\mathrm{C}-\mathrm{H}=0.93 \AA$ and $U_{\text {iso }}(\mathrm{H})=1.2 U_{\text {eq }}(\mathrm{C})$, whereas these hydrogen atoms were refined with $\mathrm{C}-\mathrm{H}=$ $0.95 \AA$ and $U_{\text {iso }}(\mathrm{H})=1.2 U_{\text {eq }}(\mathrm{C})$ for $(\mathrm{I} b)$.

For (II), the amine hydrogen atoms of the non-coordinating 4,5-dimethylbenzene-1,2-diamine were refined freely, including the isotropic displacement parameters. For the hydrogen atoms of the coordinating amines, the atomic coordinates were refined freely with $U_{\text {iso }}(\mathrm{H})=1.2 U_{\text {eq }}(\mathrm{N})$. The hydrogen atoms of the water ligands were refined freely, including the isotropic displacement parameters. The methyl hydrogen atoms were refined with $\mathrm{C}-\mathrm{H}=0.98 \AA$ and $U_{\text {iso }}(\mathrm{H})$ $=1.5 U_{\mathrm{eq}}(\mathrm{C})$.

\section{Acknowledgements}

This work was supported by a Congressionally-directed grant from the US Department of Education (grant No. P116Z100020) for the X-ray diffractometer and a grant from the Geneseo Foundation.

\section{References}

Bruker (2013). APEX2, SAINT and SADABS. Bruker AXS Inc., Madison, Wisconsin, USA.

DeStefano, M. R. \& Geiger, D. K. (2016). Acta Cryst. C72, 491-497.

Gallardo, H., Molin, F., Bortoluzzi, A. J. \& Neves, A. (2008). Acta Cryst. E64, m541-m542.

Geiger, D. K. (2012). Acta Cryst. E68, m1040.

Geiger, D. K. \& Parsons, D. E. (2014). Acta Cryst. E70, m247-m248. Grothe, E., Meekes, H., Vlieg, E., ter Horst, J. H. \& de Gelder, R. (2016). Cryst. Growth Des.16, doi: 10.1021/acs. cgd. 6b00200.

İbrahim, K., Şahin, O., Filiz, Y. \& Büyükgüngör, O. (2006). Acta Cryst. E62, m1909-m1911.

Karimnejad, K., Khaledi, H. \& Mohd Ali, H. (2011). Acta Cryst. E67, $\mathrm{m} 421$.

Lee, N. Y., Yoon, J. U. \& Jeong, J. H. (2007). Acta Cryst. E63, m2471. 
Li, F., Ou, X.-P. \& Huang, C.-C. (2012). Acta Cryst. E68, m653-m654. Lian, Z., Zhao, N., Zhang, J., Gu, Y., Li, X. \& Tang, B. (2009). Z. Kristallogr. New Cryst. Struct. 224, 399-401.

Liu, H.-Y., Wu, H., Ma, J.-F., Liu, Y.-Y., Liu, B. \& Yang, J. (2010). Cryst. Growth Des. 10, 4795-4805.

Macrae, C. F., Edgington, P. R., McCabe, P., Pidcock, E., Shields, G. P., Taylor, R., Towler, M. \& van de Streek, J. (2006). J. Appl. Cryst. 39, $453-457$.

Mei, L., Li, J., Ming, Z. S., Rong, L. Q. \& Liang, L. X. (2009). Russ. J. Coord. Chem. 35, 871-873.

Necefoglu, H., Clegg, W. \& Scott, A. J. (2001). Acta Cryst. E57, m462$\mathrm{m} 464$.

Ovalle-Marroquín, P., Gómez-Lara, J. \& Hernández-Ortega, S. (2002). Acta Cryst. E58, m269-m271.

Qian, B., Ma, W.-X., Lu, L.-D., Yang, X.-J. \& Wang, X. (2007). Acta Cryst. E63, m2930.

Sheldrick, G. M. (2008). Acta Cryst. A64, 112-122.
Sheldrick, G. M. (2015). Acta Cryst. C71, 3-8.

Shi, Y.-F., Feng, Q.-H., Zhao, W.-J., Shi, Y.-B. \& Zhan, P. (2010). Acta Cryst. E66, m593.

Singh, K., Kumar, A., Srivastava, R., Kadyan, P. S., Kamalasanan, M. N. \& Singh, I. (2011). Opt. Mater. 34, 221-227.

Spek, A. L. (2009). Acta Cryst. D65, 148-155.

Tan, R., Wang, Z.-B., Li, Y., Kozera, D. J., Lu, Z.-H. \& Song, D. (2012). Inorg. Chem. 51, 7039-7049.

Wang, C.-J., Yue, K.-F., Zhang, W.-H., Jin, J.-C., Huang, X.-Y. \& Wang, Y.-Y. (2010). Inorg. Chem. Commun. 13, 1332-1336.

Westrip, S. P. (2010). J. Appl. Cryst. 43, 920-925.

Xu, H., Xu, Z.-F., Yue, Z.-Y., Yan, P.-F., Wang, B., Jia, L.-W., Li, G.-M., Sun, W.-B. \& Zhang, J.-W. (2008). J. Phys. Chem. C, 112, 1551715525.

You, Z.-L. (2005). Acta Cryst. C61, m383-m385.

Yue, S.-M., Xu, H.-B., Ma, J.-F., Su, Z.-M., Kan, Y.-H. \& Zhang, H.-J. (2006). Polyhedron, 25, 635-644. 


\section{supporting information}

Acta Cryst. (2016). E72, 1037-1042［https://doi.org/10.1107/S2056989016010033]

\section{Structural characterization of two benzene-1,2-diamine complexes of zinc chloride: a molecular compound and a co-crystal salt}

\section{Patricia L. Zick and David K. Geiger}

Computing details

For all compounds, data collection: APEX2 (Bruker, 2013); cell refinement: SAINT (Bruker, 2013); data reduction: SAINT (Bruker, 2013); program(s) used to solve structure: SHELXS97 (Sheldrick, 2008); program(s) used to refine structure: SHELXL2014 (Sheldrick, 2015); molecular graphics: PLATON (Spek, 2009) and Mercury (Macrae et al., 2006); software used to prepare material for publication: publCIF (Westrip, 2010).

(la) (Benzene-1,2-diamine- $\left.\kappa^{2} N, N^{\prime}\right)$ dichloroidozinc

\section{Crystal data}

$\left[\mathrm{ZnCl}_{2}\left(\mathrm{C}_{6} \mathrm{H}_{8} \mathrm{~N}_{2}\right)\right]$

$M_{r}=244.41$

Orthorhombic, Pnma

$a=8.4039(9) \AA$

$b=7.5206(7) \AA$

$c=14.1667(15) \AA$

$V=895.37(16) \AA^{3}$

$Z=4$

$F(000)=488$

\section{Data collection}

Bruker SMART X2S benchtop diffractometer

Radiation source: sealed microfocus tube

Doubly curved silicon crystal monochromator

Detector resolution: 8.3330 pixels $\mathrm{mm}^{-1}$

/w scans

Absorption correction: multi-scan

(SADABS; Bruker, 2013)

$T_{\min }=0.39, T_{\max }=0.56$

Refinement

Refinement on $F^{2}$

Least-squares matrix: full

$R\left[F^{2}>2 \sigma\left(F^{2}\right)\right]=0.020$

$w R\left(F^{2}\right)=0.051$

$S=1.09$

1129 reflections

72 parameters

0 restraints

Primary atom site location: structure-invariant direct methods
$D_{\mathrm{x}}=1.813 \mathrm{Mg} \mathrm{m}^{-3}$

Mo $K \alpha$ radiation, $\lambda=0.71073 \AA$

Cell parameters from 6300 reflections

$\theta=2.8-29.2^{\circ}$

$\mu=3.27 \mathrm{~mm}^{-1}$

$T=300 \mathrm{~K}$

Parallelpiped, colorless

$0.60 \times 0.30 \times 0.20 \mathrm{~mm}$

9435 measured reflections

1129 independent reflections

1026 reflections with $I>2 \sigma(I)$

$R_{\text {int }}=0.039$

$\theta_{\max }=27.9^{\circ}, \theta_{\min }=2.8^{\circ}$

$h=-11 \rightarrow 11$

$k=-9 \rightarrow 8$

$l=-18 \rightarrow 15$

Secondary atom site location: difference Fourier map

Hydrogen site location: mixed

$\mathrm{H}$ atoms treated by a mixture of independent and constrained refinement

$w=1 /\left[\sigma^{2}\left(F_{\mathrm{o}}^{2}\right)+(0.0226 P)^{2}+0.2253 P\right]$ where $P=\left(F_{\mathrm{o}}^{2}+2 F_{\mathrm{c}}^{2}\right) / 3$

$(\Delta / \sigma)_{\max }=0.001$

$\Delta \rho_{\max }=0.26 \mathrm{e}^{-3}$

$\Delta \rho_{\min }=-0.40$ e $\AA^{-3}$ 


\section{Special details}

Geometry. All esds (except the esd in the dihedral angle between two 1.s. planes) are estimated using the full covariance matrix. The cell esds are taken into account individually in the estimation of esds in distances, angles and torsion angles; correlations between esds in cell parameters are only used when they are defined by crystal symmetry. An approximate (isotropic) treatment of cell esds is used for estimating esds involving l.s. planes.

Refinement. Refined as a 2-component inversion twin.

Fractional atomic coordinates and isotropic or equivalent isotropic displacement parameters $\left(\AA^{2}\right)$

\begin{tabular}{lllll}
\hline & $x$ & $y$ & $z$ & $U_{\text {iso }} * U_{\text {eq }}$ \\
\hline Zn1 & $0.12910(3)$ & 0.75 & $0.38035(2)$ & $0.03301(10)$ \\
$\mathrm{N} 1$ & $0.3667(2)$ & 0.75 & $0.34928(13)$ & $0.0359(5)$ \\
$\mathrm{H} 1$ & $0.393(2)$ & $0.656(3)$ & $0.3182(15)$ & $0.050(6)^{*}$ \\
$\mathrm{~N} 2$ & $0.1999(2)$ & 0.75 & $0.51849(13)$ & $0.0362(5)$ \\
$\mathrm{H} 2$ & $0.162(3)$ & $0.660(3)$ & $0.5468(16)$ & $0.057(6)^{*}$ \\
$\mathrm{C} 11$ & $0.00181(5)$ & $0.50621(6)$ & $0.33277(3)$ & $0.04250(13)$ \\
$\mathrm{C} 1$ & $0.4560(3)$ & 0.75 & $0.43712(14)$ & $0.0301(4)$ \\
$\mathrm{C} 2$ & $0.3724(2)$ & 0.75 & $0.52146(14)$ & $0.0293(4)$ \\
$\mathrm{C} 3$ & $0.4541(3)$ & 0.75 & $0.60645(15)$ & $0.0406(5)$ \\
$\mathrm{H} 3$ & 0.3982 & 0.75 & 0.6631 & $0.049^{*}$ \\
$\mathrm{C} 4$ & $0.6191(3)$ & 0.75 & $0.60699(19)$ & $0.0496(7)$ \\
$\mathrm{H} 4$ & 0.6742 & 0.75 & 0.6639 & $0.059^{*}$ \\
$\mathrm{C} 5$ & $0.7007(3)$ & 0.75 & $0.5231(2)$ & $0.0508(7)$ \\
H5 & 0.8114 & 0.75 & 0.5236 & $0.061^{*}$ \\
C6 & $0.6208(3)$ & 0.75 & $0.43830(19)$ & $0.0434(6)$ \\
H6 & 0.6774 & 0.75 & 0.3819 & $0.052^{*}$ \\
& & & &
\end{tabular}

Atomic displacement parameters $\left(\AA^{2}\right)$

\begin{tabular}{lllllll}
\hline & $U^{11}$ & $U^{22}$ & $U^{33}$ & $U^{12}$ & $U^{13}$ & $U^{23}$ \\
\hline $\mathrm{Zn} 1$ & $0.03143(15)$ & $0.04349(19)$ & $0.02409(14)$ & 0 & $-0.00277(9)$ & 0 \\
$\mathrm{~N} 1$ & $0.0353(10)$ & $0.0523(14)$ & $0.0200(8)$ & 0 & $0.0028(7)$ & 0 \\
$\mathrm{~N} 2$ & $0.0357(10)$ & $0.0506(14)$ & $0.0223(8)$ & 0 & $0.0037(7)$ & 0 \\
$\mathrm{C} 11$ & $0.0528(3)$ & $0.0391(3)$ & $0.0355(2)$ & $-0.00706(18)$ & $-0.00622(16)$ & $0.00356(16)$ \\
$\mathrm{C} 1$ & $0.0339(11)$ & $0.0307(12)$ & $0.0258(9)$ & 0 & $0.0001(8)$ & 0 \\
$\mathrm{C} 2$ & $0.0342(11)$ & $0.0308(12)$ & $0.0228(9)$ & 0 & $-0.0010(7)$ & 0 \\
$\mathrm{C} 3$ & $0.0499(14)$ & $0.0474(15)$ & $0.0244(10)$ & 0 & $-0.0057(9)$ & 0 \\
$\mathrm{C} 4$ & $0.0485(15)$ & $0.0559(18)$ & $0.0443(14)$ & 0 & $-0.0201(11)$ & 0 \\
$\mathrm{C} 5$ & $0.0354(13)$ & $0.0582(18)$ & $0.0587(15)$ & 0 & $-0.0093(11)$ & 0 \\
$\mathrm{C} 6$ & $0.0332(12)$ & $0.0541(17)$ & $0.0429(13)$ & 0 & $0.0041(9)$ & 0 \\
\hline
\end{tabular}

Geometric parameters $\left(\AA,{ }^{\circ}\right)$

\begin{tabular}{llll}
\hline $\mathrm{Zn} 1-\mathrm{C} 11^{\mathrm{i}}$ & $2.2271(5)$ & $\mathrm{C} 5-\mathrm{C} 6$ & $1.377(4)$ \\
$\mathrm{Zn} 1-\mathrm{C} 11$ & $2.2271(5)$ & $\mathrm{C} 5-\mathrm{H} 5$ & 0.93 \\
$\mathrm{Zn} 1-\mathrm{N} 1$ & $2.0449(19)$ & $\mathrm{C} 4-\mathrm{C} 5$ & $1.372(4)$ \\
$\mathrm{Zn} 1-\mathrm{N} 2$ & $2.0454(18)$ & $\mathrm{C} 4-\mathrm{H} 4$ & 0.93 \\
$\mathrm{~N} 2-\mathrm{C} 2$ & $1.451(3)$ & $\mathrm{C} 3-\mathrm{C} 4$ & $1.387(4)$
\end{tabular}




$\begin{array}{llll}\mathrm{N} 2-\mathrm{H} 2 & 0.85(2) & \mathrm{C} 3-\mathrm{H} 3 & 0.93 \\ \mathrm{~N} 1-\mathrm{C} 1 & 1.453(3) & \mathrm{C} 2-\mathrm{C} 3 & 1.386(3) \\ \mathrm{N} 1-\mathrm{H} 1 & 0.86(2) & \mathrm{C} 1-\mathrm{C} 2 & 1.386(3) \\ \mathrm{C} 6-\mathrm{H} 6 & 0.93 & \mathrm{C} 1-\mathrm{C} 6 & 1.385(3) \\ & & & \\ \mathrm{C} 11-\mathrm{Zn} 1-\mathrm{C} 11^{\mathrm{i}} & 110.82(2) & \mathrm{C} 4-\mathrm{C} 5-\mathrm{C} 6 & 120.8(2) \\ \mathrm{N} 1-\mathrm{Zn} 1-\mathrm{N} 2 & 85.53(8) & \mathrm{C} 6-\mathrm{C} 5-\mathrm{H} 5 & 119.6 \\ \mathrm{~N} 1-\mathrm{Zn} 1-\mathrm{C} 11 & \mathrm{C} 4-\mathrm{C} 5-\mathrm{H} 5 & 119.6 \\ \mathrm{~N} 2-\mathrm{Zn} 1-\mathrm{C} 11 & \mathrm{C} 5-\mathrm{C} 4-\mathrm{C} 3 & 119.7(2) \\ \mathrm{N} 2-\mathrm{Zn} 1-\mathrm{C} 11^{\mathrm{i}} & 113.82(3) & \mathrm{C} 5-\mathrm{C} 4-\mathrm{H} 4 & 120.2 \\ \mathrm{~N} 1-\mathrm{Zn} 1-\mathrm{C} 11^{\mathrm{i}} & 115.42(3) & \mathrm{C} 3-\mathrm{C} 4-\mathrm{H} 4 & 120.2 \\ \mathrm{Zn} 1-\mathrm{N} 2-\mathrm{H} 2 & 113.82(3) & \mathrm{C} 2-\mathrm{C} 3-\mathrm{C} 4 & 120.0(2) \\ \mathrm{C} 2-\mathrm{N} 2-\mathrm{H} 2 & 110.1(15) & \mathrm{C} 4-\mathrm{C} 3-\mathrm{H} 3 & 120.0 \\ \mathrm{C} 2-\mathrm{N} 2-\mathrm{Zn} 1 & 111.3(15) & \mathrm{C} 2-\mathrm{C} 3-\mathrm{H} 3 & 119.8(2) \\ \mathrm{Zn} 1-\mathrm{N} 1-\mathrm{H} 1 & 108.56(13) & \mathrm{C} 3-\mathrm{C} 2-\mathrm{C} 1 & 121.4(2) \\ \mathrm{C} 1-\mathrm{N} 1-\mathrm{H} 1 & 111.1(14) & \mathrm{C} 3-\mathrm{C} 2-\mathrm{N} 2 & 118.81(18) \\ \mathrm{C} 1-\mathrm{N} 1-\mathrm{Zn} 1 & 107.8(14) & \mathrm{C} 1-\mathrm{C} 2-\mathrm{N} 2 & 119.8(2) \\ \mathrm{C} 5-\mathrm{C} 6-\mathrm{C} 1 & 108.67(13) & \mathrm{C} 6-\mathrm{C} 1-\mathrm{C} 2 & 121.79(19) \\ \mathrm{C} 5-\mathrm{C} 6-\mathrm{H} 6 & 119.9(2) & \mathrm{C} 6-\mathrm{C} 1-\mathrm{N} 1 & 118.43(19) \\ \mathrm{C} 1-\mathrm{C} 6-\mathrm{H} 6 & 120.1 & \mathrm{C} 2-\mathrm{C} 1-\mathrm{N} 1 & \\ & 120.1 & & \end{array}$

Symmetry code: (i) $x,-y+3 / 2, z$.

Hydrogen-bond geometry $\left(\AA,{ }^{\circ}\right)$

\begin{tabular}{lllll}
\hline$D-\mathrm{H} \cdots A$ & $D-\mathrm{H}$ & $\mathrm{H} \cdots A$ & $D \cdots A$ & $D-\mathrm{H} \cdots A$ \\
\hline $\mathrm{N} 1-\mathrm{H} 1 \cdots \mathrm{C} 1{ }^{1 i}$ & $0.86(2)$ & $2.59(2)$ & $3.3618(16)$ & $150.7(18)$ \\
$\mathrm{N} 2-\mathrm{H} 2 \cdots \mathrm{Cl} 1^{\mathrm{iii}}$ & $0.85(2)$ & $2.52(2)$ & $3.3204(16)$ & $157(2)$
\end{tabular}

Symmetry codes: (ii) $x+1 / 2, y,-z+1 / 2$; (iii) $-x,-y+1,-z+1$.

(II) trans-Diaquabis(4,5-dimethylbenzene-1,2-diamine- $\left.\kappa^{2} N, N^{\prime}\right)$ zinc chloride-4,5-dimethylbenzene-1,2-diamine

Crystal data

$\left[\mathrm{Zn}\left(\mathrm{C}_{8} \mathrm{H}_{12} \mathrm{~N}_{2}\right)_{2}\left(\mathrm{H}_{2} \mathrm{O}\right)_{2}\right] \mathrm{Cl}_{2} \cdot 2 \mathrm{C}_{8} \mathrm{H}_{12} \mathrm{~N}_{2}$

$M_{r}=717.08$

Monoclinic, $P 2{ }_{1} / c$

$a=18.529(2) \AA$

$b=12.6227(16) \AA$

$c=7.8691(8) \AA$

$\beta=94.665(4)^{\circ}$

$V=1834.4(4) \AA^{3}$

$Z=2$

$F(000)=760$

$D_{\mathrm{x}}=1.298 \mathrm{Mg} \mathrm{m}^{-3}$

Mo $K \alpha$ radiation, $\lambda=0.71073 \AA$

Cell parameters from 8719 reflections

$\theta=2.7-25.8^{\circ}$

$\mu=0.86 \mathrm{~mm}^{-1}$

$T=200 \mathrm{~K}$

Plate, clear colourless

$0.60 \times 0.40 \times 0.10 \mathrm{~mm}$

\section{Data collection}

Bruker SMART X2S benchtop diffractometer

Radiation source: sealed microfocus tube

Doubly curved silicon crystal monochromator

Detector resolution: 8.3330 pixels $\mathrm{mm}^{-1}$

/w scans
Absorption correction: multi-scan

(SADABS; Bruker, 2013)

$T_{\min }=0.66, T_{\max }=0.92$

25430 measured reflections

3619 independent reflections

2920 reflections with $I>2 \sigma(I)$ 


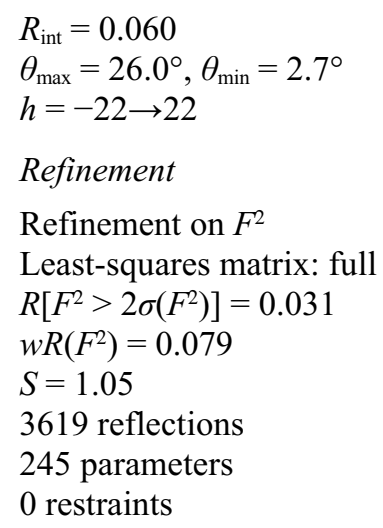

Refinement

Refinement on $F^{2}$

Least-squares matrix: full

$R\left[F^{2}>2 \sigma\left(F^{2}\right)\right]=0.031$

$w R\left(F^{2}\right)=0.079$

$S=1.05$

3619 reflections

245 parameters

0 restraints

$$
\begin{aligned}
& k=-15 \rightarrow 15 \\
& l=-9 \rightarrow 9
\end{aligned}
$$

Hydrogen site location: mixed

$\mathrm{H}$ atoms treated by a mixture of independent and constrained refinement

$w=1 /\left[\sigma^{2}\left(F_{\mathrm{o}}^{2}\right)+(0.0325 P)^{2}+0.4174 P\right]$ where $P=\left(F_{\mathrm{o}}^{2}+2 F_{\mathrm{c}}{ }^{2}\right) / 3$

$(\Delta / \sigma)_{\max }=0.001$

$\Delta \rho_{\max }=0.31$ e $\AA^{-3}$

$\Delta \rho_{\min }=-0.22$ e $\AA^{-3}$

Special details

Geometry. All esds (except the esd in the dihedral angle between two 1.s. planes) are estimated using the full covariance matrix. The cell esds are taken into account individually in the estimation of esds in distances, angles and torsion angles; correlations between esds in cell parameters are only used when they are defined by crystal symmetry. An approximate (isotropic) treatment of cell esds is used for estimating esds involving l.s. planes.

\begin{tabular}{|c|c|c|c|c|}
\hline & $x$ & $y$ & $z$ & $U_{\text {iso }} * / U_{\text {eq }}$ \\
\hline $\mathrm{Zn} 1$ & 0.5 & 0.5 & 0.5 & $0.02953(11)$ \\
\hline $\mathrm{Cl1}$ & $0.45668(3)$ & $0.33892(4)$ & $1.02374(6)$ & $0.03564(13)$ \\
\hline $\mathrm{O} 1$ & $0.56720(9)$ & $0.42255(13)$ & 0.31090 (19) & $0.0392(4)$ \\
\hline H1WA & $0.6043(14)$ & $0.447(2)$ & $0.278(3)$ & $0.051(8)^{*}$ \\
\hline H1WB & $0.5410(14)$ & $0.403(2)$ & $0.231(3)$ & $0.058(8)^{*}$ \\
\hline N1 & $0.40508(8)$ & $0.41964(13)$ & $0.4016(2)$ & $0.0274(3)$ \\
\hline H1A & $0.4163(11)$ & $0.3837(17)$ & $0.317(3)$ & $0.033^{*}$ \\
\hline H1B & $0.3870(11)$ & $0.3749(17)$ & $0.468(3)$ & $0.033^{*}$ \\
\hline N2 & $0.45593(9)$ & $0.61470(14)$ & $0.3192(2)$ & $0.0322(4)$ \\
\hline $\mathrm{H} 2 \mathrm{~A}$ & $0.4637(11)$ & $0.6792(18)$ & $0.355(3)$ & $0.039 *$ \\
\hline $\mathrm{H} 2 \mathrm{~B}$ & $0.4755(12)$ & $0.6043(17)$ & $0.233(3)$ & $0.039 *$ \\
\hline N3 & $0.67491(11)$ & $0.80052(17)$ & $0.8184(3)$ & $0.0422(4)$ \\
\hline H3A & $0.6415(14)$ & $0.807(2)$ & $0.741(3)$ & $0.056(8)^{*}$ \\
\hline H3B & $0.6781(12)$ & $0.736(2)$ & 0.859 (3) & $0.050(7)^{*}$ \\
\hline N4 & 0.68407 (11) & $0.98800(18)$ & $0.6276(3)$ & $0.0408(4)$ \\
\hline $\mathrm{H} 4 \mathrm{~A}$ & $0.6917(13)$ & $1.035(2)$ & $0.560(3)$ & $0.058(8)^{*}$ \\
\hline H4B & 0.6509 (14) & $0.947(2)$ & $0.579(3)$ & $0.053(7)^{*}$ \\
\hline $\mathrm{C} 1$ & $0.35297(10)$ & $0.49852(14)$ & $0.3405(2)$ & $0.0261(4)$ \\
\hline $\mathrm{C} 2$ & $0.37896(10)$ & $0.59711(15)$ & $0.2950(2)$ & $0.0280(4)$ \\
\hline $\mathrm{C} 3$ & $0.33004(11)$ & $0.67499(16)$ & $0.2373(2)$ & $0.0356(5)$ \\
\hline H3 & 0.3479 & 0.7416 & 0.2031 & $0.043^{*}$ \\
\hline $\mathrm{C} 4$ & $0.25549(11)$ & $0.65820(17)$ & $0.2281(2)$ & $0.0385(5)$ \\
\hline $\mathrm{C} 5$ & $0.22942(10)$ & $0.56000(18)$ & $0.2798(2)$ & $0.0373(5)$ \\
\hline C6 & $0.27863(10)$ & $0.48108(16)$ & $0.3337(2)$ & $0.0325(5)$ \\
\hline H6 & 0.2611 & 0.414 & 0.3666 & $0.039 *$ \\
\hline $\mathrm{C} 7$ & $0.20454(14)$ & $0.7463(2)$ & 0.1635 & $0.0584(7)$ \\
\hline $\mathrm{H} 7 \mathrm{~A}$ & 0.2328 & 0.8085 & 0.1351 & $0.088^{*}$ \\
\hline
\end{tabular}

Fractional atomic coordinates and isotropic or equivalent isotropic displacement parameters $\left(\AA^{2}\right)$ 


$\begin{array}{lllll}\text { H7B } & 0.1726 & 0.7651 & 0.2522 & 0.088^{*} \\ \text { H7C } & 0.1753 & 0.7221 & 0.0615 & 0.088^{*} \\ \text { C8 } & 0.14910(12) & 0.5376(2) & 0.2831(3) & 0.0575(7) \\ \text { H8A } & 0.1248 & 0.5496 & 0.1694 & 0.086^{*} \\ \text { H8B } & 0.1285 & 0.5849 & 0.3653 & 0.086^{*} \\ \text { H8C } & 0.1421 & 0.4638 & 0.3168 & 0.086^{*} \\ \text { C9 } & 0.74336(10) & 0.84050(16) & 0.7842(2) & 0.0337(4) \\ \text { C10 } & 0.74828(10) & 0.93442(16) & 0.6917(2) & 0.0330(4) \\ \text { C11 } & 0.81603(12) & 0.97694(17) & 0.6739(3) & 0.0389(5) \\ \text { H11 } & 0.8193 & 1.0404 & 0.61 & 0.047^{*} \\ \text { C12 } & 0.87968(11) & 0.93093(19) & 0.7455(3) & 0.0437(5) \\ \text { C13 } & 0.87500(12) & 0.83640(19) & 0.8363(3) & 0.0441(5) \\ \text { C14 } & 0.80689(12) & 0.79322(18) & 0.8537(2) & 0.0403(5) \\ \text { H14 } & 0.8036 & 0.7289 & 0.9154 & 0.048^{*} \\ \text { C15 } & 0.95146(14) & 0.9844(2) & 0.7237(4) & 0.0706(8) \\ \text { H15A } & 0.9767 & 0.9977 & 0.836 & 0.106^{*} \\ \text { H15B } & 0.9429 & 1.0518 & 0.6636 & 0.106^{*} \\ \text { H15C } & 0.9812 & 0.9384 & 0.6575 & 0.106^{*} \\ \text { C16 } & 0.94172(14) & 0.7809(3) & 0.9159(3) & 0.0710(8) \\ \text { H16A } & 0.9745 & 0.7651 & 0.8275 & 0.107^{*} \\ \text { H16B } & 0.9275 & 0.7147 & 0.9691 & 0.107^{*} \\ \text { H16C } & 0.9664 & 0.8268 & 1.0026 & 0.107^{*}\end{array}$

Atomic displacement parameters $\left(\AA^{2}\right)$

\begin{tabular}{lllllll}
\hline & $U^{11}$ & $U^{22}$ & $U^{33}$ & $U^{12}$ & $U^{13}$ & $U^{23}$ \\
\hline Zn1 & $0.02417(16)$ & $0.03331(19)$ & $0.03039(18)$ & $-0.00446(13)$ & $-0.00213(12)$ & $-0.00003(13)$ \\
C11 & $0.0358(3)$ & $0.0413(3)$ & $0.0299(3)$ & $-0.0041(2)$ & $0.00360(19)$ & $0.0004(2)$ \\
O1 & $0.0308(8)$ & $0.0510(10)$ & $0.0362(9)$ & $-0.0069(7)$ & $0.0048(7)$ & $-0.0091(7)$ \\
N1 & $0.0275(8)$ & $0.0270(9)$ & $0.0280(9)$ & $-0.0030(7)$ & $0.0028(7)$ & $-0.0012(7)$ \\
N2 & $0.0310(9)$ & $0.0344(9)$ & $0.0318(9)$ & $-0.0076(7)$ & $0.0061(7)$ & $0.0018(8)$ \\
N3 & $0.0411(11)$ & $0.0428(12)$ & $0.0436(11)$ & $-0.0114(9)$ & $0.0094(9)$ & $-0.0012(9)$ \\
N4 & $0.0359(10)$ & $0.0443(12)$ & $0.0422(11)$ & $-0.0001(9)$ & $0.0027(8)$ & $0.0053(10)$ \\
C1 & $0.0261(9)$ & $0.0311(10)$ & $0.0209(9)$ & $-0.0002(8)$ & $0.0004(7)$ & $-0.0021(8)$ \\
C2 & $0.0287(9)$ & $0.0329(10)$ & $0.0225(9)$ & $-0.0024(8)$ & $0.0019(7)$ & $0.0002(8)$ \\
C3 & $0.0435(11)$ & $0.0314(11)$ & $0.0318(11)$ & $0.0007(9)$ & $0.0024(9)$ & $0.0035(8)$ \\
C4 & $0.0401(11)$ & $0.0471(13)$ & $0.0279(10)$ & $0.0131(10)$ & $0.0005(8)$ & $-0.0020(9)$ \\
C5 & $0.0279(10)$ & $0.0544(14)$ & $0.0297(10)$ & $0.0019(9)$ & $0.0020(8)$ & $-0.0012(9)$ \\
C6 & $0.0273(10)$ & $0.0400(12)$ & $0.0301(10)$ & $-0.0055(8)$ & $0.0024(8)$ & $0.0004(8)$ \\
C7 & $0.0582(15)$ & $0.0603(17)$ & $0.0559(15)$ & $0.0239(13)$ & $-0.0010(12)$ & $0.0009(12)$ \\
C8 & $0.0301(12)$ & $0.0844(19)$ & $0.0582(15)$ & $0.0066(12)$ & $0.0043(11)$ & $0.0051(13)$ \\
C9 & $0.0359(11)$ & $0.0376(12)$ & $0.0288(10)$ & $-0.0068(9)$ & $0.0093(8)$ & $-0.0070(9)$ \\
C10 & $0.0354(11)$ & $0.0364(11)$ & $0.0276(10)$ & $-0.0011(9)$ & $0.0060(8)$ & $-0.0047(8)$ \\
C11 & $0.0405(12)$ & $0.0393(12)$ & $0.0379(11)$ & $-0.0076(9)$ & $0.0089(9)$ & $0.0030(9)$ \\
C12 & $0.0342(11)$ & $0.0583(15)$ & $0.0394(12)$ & $-0.0083(10)$ & $0.0071(9)$ & $-0.0055(11)$ \\
C13 & $0.0389(12)$ & $0.0572(15)$ & $0.0361(11)$ & $0.0042(11)$ & $0.0025(9)$ & $-0.0024(10)$ \\
C14 & $0.0493(13)$ & $0.0380(12)$ & $0.0342(11)$ & $0.0031(10)$ & $0.0069(9)$ & $0.0025(9)$ \\
C15 & $0.0383(14)$ & $0.100(2)$ & $0.0741(19)$ & $-0.0179(14)$ & $0.0070(13)$ & $0.0068(16)$ \\
& & & & & &
\end{tabular}


$\begin{array}{lllllll}\mathrm{C} 16 & 0.0503(15) & 0.095(2) & 0.0656(17) & 0.0143(15) & -0.0073(13) & 0.0084(16)\end{array}$

Geometric parameters $(\AA, \stackrel{o}{)}$

\begin{tabular}{|c|c|c|c|}
\hline $\mathrm{Zn} 1-\mathrm{N} 1^{\mathrm{i}}$ & $2.1214(15)$ & $\mathrm{C} 4-\mathrm{C} 7$ & $1.519(3)$ \\
\hline $\mathrm{Zn} 1-\mathrm{N} 1$ & $2.1214(15)$ & $\mathrm{C} 5-\mathrm{C} 6$ & $1.393(3)$ \\
\hline $\mathrm{Zn} 1-\mathrm{N} 2$ & $2.1442(17)$ & $\mathrm{C} 5-\mathrm{C} 8$ & $1.517(3)$ \\
\hline $\mathrm{Zn} 1-\mathrm{N} 2^{\mathrm{i}}$ & $2.1442(17)$ & C6- 6 6 & 0.95 \\
\hline $\mathrm{Zn} 1-\mathrm{O}^{\mathrm{i}}$ & $2.2409(15)$ & C7-H7A & 0.98 \\
\hline $\mathrm{Zn} 1-\mathrm{O} 1$ & $2.2410(15)$ & C7-H7B & 0.98 \\
\hline $\mathrm{O} 1-\mathrm{H} 1 \mathrm{WA}$ & $0.81(3)$ & $\mathrm{C} 7-\mathrm{H} 7 \mathrm{C}$ & 0.98 \\
\hline $\mathrm{O} 1-\mathrm{H} 1 \mathrm{WB}$ & $0.80(3)$ & $\mathrm{C} 8-\mathrm{H} 8 \mathrm{~A}$ & 0.98 \\
\hline $\mathrm{N} 1-\mathrm{C} 1$ & $1.442(2)$ & $\mathrm{C} 8-\mathrm{H} 8 \mathrm{~B}$ & 0.98 \\
\hline $\mathrm{N} 1-\mathrm{H} 1 \mathrm{~A}$ & $0.84(2)$ & $\mathrm{C} 8-\mathrm{H} 8 \mathrm{C}$ & 0.98 \\
\hline N1-H1B & $0.85(2)$ & $\mathrm{C} 9-\mathrm{C} 14$ & $1.391(3)$ \\
\hline $\mathrm{N} 2-\mathrm{C} 2$ & $1.441(2)$ & $\mathrm{C} 9-\mathrm{C} 10$ & $1.398(3)$ \\
\hline $\mathrm{N} 2-\mathrm{H} 2 \mathrm{~A}$ & $0.87(2)$ & $\mathrm{C} 10-\mathrm{C} 11$ & $1.383(3)$ \\
\hline $\mathrm{N} 2-\mathrm{H} 2 \mathrm{~B}$ & $0.81(2)$ & $\mathrm{C} 11-\mathrm{C} 12$ & $1.392(3)$ \\
\hline $\mathrm{N} 3-\mathrm{C} 9$ & $1.411(3)$ & $\mathrm{C} 11-\mathrm{H} 11$ & 0.95 \\
\hline $\mathrm{N} 3-\mathrm{H} 3 \mathrm{~A}$ & $0.84(3)$ & $\mathrm{C} 12-\mathrm{C} 13$ & $1.397(3)$ \\
\hline N3-H3B & $0.87(3)$ & $\mathrm{C} 12-\mathrm{C} 15$ & $1.514(3)$ \\
\hline $\mathrm{N} 4-\mathrm{C} 10$ & $1.425(3)$ & $\mathrm{C} 13-\mathrm{C} 14$ & $1.392(3)$ \\
\hline $\mathrm{N} 4-\mathrm{H} 4 \mathrm{~A}$ & $0.81(3)$ & $\mathrm{C} 13-\mathrm{C} 16$ & $1.512(3)$ \\
\hline N4-H4B & $0.87(3)$ & $\mathrm{C} 14-\mathrm{H} 14$ & 0.95 \\
\hline $\mathrm{C} 1-\mathrm{C} 6$ & $1.392(2)$ & $\mathrm{C} 15-\mathrm{H} 15 \mathrm{~A}$ & 0.98 \\
\hline $\mathrm{C} 1-\mathrm{C} 2$ & $1.392(3)$ & C15-H15B & 0.98 \\
\hline $\mathrm{C} 2-\mathrm{C} 3$ & $1.388(3)$ & $\mathrm{C} 15-\mathrm{H} 15 \mathrm{C}$ & 0.98 \\
\hline $\mathrm{C} 3-\mathrm{C} 4$ & $1.393(3)$ & $\mathrm{C} 16-\mathrm{H} 16 \mathrm{~A}$ & 0.98 \\
\hline $\mathrm{C} 3-\mathrm{H} 3$ & 0.95 & $\mathrm{C} 16-\mathrm{H} 16 \mathrm{~B}$ & 0.98 \\
\hline $\mathrm{C} 4-\mathrm{C} 5$ & $1.403(3)$ & $\mathrm{C} 16-\mathrm{H} 16 \mathrm{C}$ & 0.98 \\
\hline $\mathrm{N} 1{ }^{\mathrm{i}-} \mathrm{Zn} 1-\mathrm{N} 1$ & 180.0 & $\mathrm{C} 6-\mathrm{C} 5-\mathrm{C} 4$ & $119.21(18)$ \\
\hline $\mathrm{N} 1{ }^{\mathrm{i}}-\mathrm{Zn} 1-\mathrm{N} 2$ & $100.31(6)$ & $\mathrm{C} 6-\mathrm{C} 5-\mathrm{C} 8$ & $118.7(2)$ \\
\hline $\mathrm{N} 1-\mathrm{Zn} 1-\mathrm{N} 2$ & $79.69(6)$ & $\mathrm{C} 4-\mathrm{C} 5-\mathrm{C} 8$ & $122.1(2)$ \\
\hline $\mathrm{N} 1^{\mathrm{i}}-\mathrm{Zn} 1-\mathrm{N} 2^{\mathrm{i}}$ & $79.69(6)$ & $\mathrm{C} 1-\mathrm{C} 6-\mathrm{C} 5$ & $121.32(19)$ \\
\hline $\mathrm{N} 1-\mathrm{Zn} 1-\mathrm{N} 2^{\mathrm{i}}$ & $100.31(6)$ & $\mathrm{C} 1-\mathrm{C} 6-\mathrm{H} 6$ & 119.3 \\
\hline $\mathrm{N} 2-\mathrm{Zn} 1-\mathrm{N} 2^{\mathrm{i}}$ & 180.0 & $\mathrm{C} 5-\mathrm{C} 6-\mathrm{H} 6$ & 119.3 \\
\hline $\mathrm{N} 1^{\mathrm{i}}-\mathrm{Zn} 1-\mathrm{O} 1^{\mathrm{i}}$ & $92.18(7)$ & $\mathrm{C} 4-\mathrm{C} 7-\mathrm{H} 7 \mathrm{~A}$ & 109.5 \\
\hline $\mathrm{N} 1-\mathrm{Zn} 1-\mathrm{O} 1^{\mathrm{i}}$ & $87.82(7)$ & $\mathrm{C} 4-\mathrm{C} 7-\mathrm{H} 7 \mathrm{~B}$ & 109.5 \\
\hline $\mathrm{N} 2-\mathrm{Zn} 1-\mathrm{O} 1^{\mathrm{i}}$ & $86.78(7)$ & $\mathrm{H} 7 \mathrm{~A}-\mathrm{C} 7-\mathrm{H} 7 \mathrm{~B}$ & 109.5 \\
\hline $\mathrm{N} 2^{\mathrm{i}}-\mathrm{Zn} 1-\mathrm{O} 1^{\mathrm{i}}$ & $93.22(7)$ & $\mathrm{C} 4-\mathrm{C} 7-\mathrm{H} 7 \mathrm{C}$ & 109.5 \\
\hline $\mathrm{N} 1-\mathrm{Zn} 1-\mathrm{O} 1$ & $87.82(7)$ & $\mathrm{H} 7 \mathrm{~A}-\mathrm{C} 7-\mathrm{H} 7 \mathrm{C}$ & 109.5 \\
\hline $\mathrm{N} 1-\mathrm{Zn} 1-\mathrm{O} 1$ & $92.18(7)$ & $\mathrm{H} 7 \mathrm{~B}-\mathrm{C} 7-\mathrm{H} 7 \mathrm{C}$ & 109.5 \\
\hline $\mathrm{N} 2-\mathrm{Zn} 1-\mathrm{O} 1$ & $93.22(7)$ & $\mathrm{C} 5-\mathrm{C} 8-\mathrm{H} 8 \mathrm{~A}$ & 109.5 \\
\hline $\mathrm{N} 22^{\mathrm{i}}-\mathrm{Zn} 1-\mathrm{O} 1$ & $86.78(7)$ & $\mathrm{C} 5-\mathrm{C} 8-\mathrm{H} 8 \mathrm{~B}$ & 109.5 \\
\hline $\mathrm{O} 1-\mathrm{Zn} 1-\mathrm{O} 1$ & $180.00(5)$ & $\mathrm{H} 8 \mathrm{~A}-\mathrm{C} 8-\mathrm{H} 8 \mathrm{~B}$ & 109.5 \\
\hline $\mathrm{Zn} 1-\mathrm{O} 1-\mathrm{H} 1 \mathrm{WA}$ & $124.6(18)$ & $\mathrm{C} 5-\mathrm{C} 8-\mathrm{H} 8 \mathrm{C}$ & 109.5 \\
\hline $\mathrm{Zn} 1-\mathrm{O} 1-\mathrm{H} 1 \mathrm{WB}$ & $108.7(18)$ & $\mathrm{H} 8 \mathrm{~A}-\mathrm{C} 8-\mathrm{H} 8 \mathrm{C}$ & 109.5 \\
\hline
\end{tabular}




\begin{tabular}{|c|c|c|c|}
\hline $\mathrm{H} 1 \mathrm{WA}-\mathrm{O} 1-\mathrm{H} 1 \mathrm{WB}$ & $110(2)$ & $\mathrm{H} 8 \mathrm{~B}-\mathrm{C} 8-\mathrm{H} 8 \mathrm{C}$ & 109.5 \\
\hline $\mathrm{C} 1-\mathrm{N} 1-\mathrm{Zn} 1$ & $107.71(12)$ & $\mathrm{C} 14-\mathrm{C} 9-\mathrm{C} 10$ & $118.65(18)$ \\
\hline $\mathrm{C} 1-\mathrm{N} 1-\mathrm{H} 1 \mathrm{~A}$ & $108.0(14)$ & $\mathrm{C} 14-\mathrm{C} 9-\mathrm{N} 3$ & $121.1(2)$ \\
\hline $\mathrm{Zn} 1-\mathrm{N} 1-\mathrm{H} 1 \mathrm{~A}$ & $107.1(14)$ & $\mathrm{C} 10-\mathrm{C} 9-\mathrm{N} 3$ & $120.01(19)$ \\
\hline $\mathrm{C} 1-\mathrm{N} 1-\mathrm{H} 1 \mathrm{~B}$ & $111.9(14)$ & $\mathrm{C} 11-\mathrm{C} 10-\mathrm{C} 9$ & $118.68(19)$ \\
\hline $\mathrm{Zn} 1-\mathrm{N} 1-\mathrm{H} 1 \mathrm{~B}$ & $116.6(13)$ & $\mathrm{C} 11-\mathrm{C} 10-\mathrm{N} 4$ & $121.26(19)$ \\
\hline $\mathrm{H} 1 \mathrm{~A}-\mathrm{N} 1-\mathrm{H} 1 \mathrm{~B}$ & $105(2)$ & $\mathrm{C} 9-\mathrm{C} 10-\mathrm{N} 4$ & $119.93(18)$ \\
\hline $\mathrm{C} 2-\mathrm{N} 2-\mathrm{Zn} 1$ & $107.66(11)$ & $\mathrm{C} 10-\mathrm{C} 11-\mathrm{C} 12$ & $122.9(2)$ \\
\hline $\mathrm{C} 2-\mathrm{N} 2-\mathrm{H} 2 \mathrm{~A}$ & $108.9(14)$ & $\mathrm{C} 10-\mathrm{C} 11-\mathrm{H} 11$ & 118.5 \\
\hline $\mathrm{Zn} 1-\mathrm{N} 2-\mathrm{H} 2 \mathrm{~A}$ & $111.8(14)$ & $\mathrm{C} 12-\mathrm{C} 11-\mathrm{H} 11$ & 118.5 \\
\hline $\mathrm{C} 2-\mathrm{N} 2-\mathrm{H} 2 \mathrm{~B}$ & $111.9(15)$ & $\mathrm{C} 11-\mathrm{C} 12-\mathrm{C} 13$ & $118.52(19)$ \\
\hline $\mathrm{Zn} 1-\mathrm{N} 2-\mathrm{H} 2 \mathrm{~B}$ & $106.0(16)$ & $\mathrm{C} 11-\mathrm{C} 12-\mathrm{C} 15$ & $119.4(2)$ \\
\hline $\mathrm{H} 2 \mathrm{~A}-\mathrm{N} 2-\mathrm{H} 2 \mathrm{~B}$ & $111(2)$ & $\mathrm{C} 13-\mathrm{C} 12-\mathrm{C} 15$ & $122.0(2)$ \\
\hline $\mathrm{C} 9-\mathrm{N} 3-\mathrm{H} 3 \mathrm{~A}$ & $116.8(17)$ & $\mathrm{C} 14-\mathrm{C} 13-\mathrm{C} 12$ & $118.65(19)$ \\
\hline $\mathrm{C} 9-\mathrm{N} 3-\mathrm{H} 3 \mathrm{~B}$ & $111.6(16)$ & $\mathrm{C} 14-\mathrm{C} 13-\mathrm{C} 16$ & $119.7(2)$ \\
\hline $\mathrm{H} 3 \mathrm{~A}-\mathrm{N} 3-\mathrm{H} 3 \mathrm{~B}$ & $112(2)$ & $\mathrm{C} 12-\mathrm{C} 13-\mathrm{C} 16$ & $121.7(2)$ \\
\hline $\mathrm{C} 10-\mathrm{N} 4-\mathrm{H} 4 \mathrm{~A}$ & $113.0(18)$ & $\mathrm{C} 9-\mathrm{C} 14-\mathrm{C} 13$ & $122.6(2)$ \\
\hline $\mathrm{C} 10-\mathrm{N} 4-\mathrm{H} 4 \mathrm{~B}$ & $114.7(17)$ & $\mathrm{C} 9-\mathrm{C} 14-\mathrm{H} 14$ & 118.7 \\
\hline $\mathrm{H} 4 \mathrm{~A}-\mathrm{N} 4-\mathrm{H} 4 \mathrm{~B}$ & $107(2)$ & $\mathrm{C} 13-\mathrm{C} 14-\mathrm{H} 14$ & 118.7 \\
\hline $\mathrm{C} 6-\mathrm{C} 1-\mathrm{C} 2$ & $119.58(17)$ & $\mathrm{C} 12-\mathrm{C} 15-\mathrm{H} 15 \mathrm{~A}$ & 109.5 \\
\hline $\mathrm{C} 6-\mathrm{C} 1-\mathrm{N} 1$ & $122.50(17)$ & $\mathrm{C} 12-\mathrm{C} 15-\mathrm{H} 15 \mathrm{~B}$ & 109.5 \\
\hline $\mathrm{C} 2-\mathrm{C} 1-\mathrm{N} 1$ & $117.84(16)$ & $\mathrm{H} 15 \mathrm{~A}-\mathrm{C} 15-\mathrm{H} 15 \mathrm{~B}$ & 109.5 \\
\hline $\mathrm{C} 3-\mathrm{C} 2-\mathrm{C} 1$ & $119.14(17)$ & $\mathrm{C} 12-\mathrm{C} 15-\mathrm{H} 15 \mathrm{C}$ & 109.5 \\
\hline $\mathrm{C} 3-\mathrm{C} 2-\mathrm{N} 2$ & $123.19(18)$ & $\mathrm{H} 15 \mathrm{~A}-\mathrm{C} 15-\mathrm{H} 15 \mathrm{C}$ & 109.5 \\
\hline $\mathrm{C} 1-\mathrm{C} 2-\mathrm{N} 2$ & $117.59(16)$ & $\mathrm{H} 15 \mathrm{~B}-\mathrm{C} 15-\mathrm{H} 15 \mathrm{C}$ & 109.5 \\
\hline $\mathrm{C} 2-\mathrm{C} 3-\mathrm{C} 4$ & $121.84(19)$ & $\mathrm{C} 13-\mathrm{C} 16-\mathrm{H} 16 \mathrm{~A}$ & 109.5 \\
\hline $\mathrm{C} 2-\mathrm{C} 3-\mathrm{H} 3$ & 119.1 & $\mathrm{C} 13-\mathrm{C} 16-\mathrm{H} 16 \mathrm{~B}$ & 109.5 \\
\hline $\mathrm{C} 4-\mathrm{C} 3-\mathrm{H} 3$ & 119.1 & $\mathrm{H} 16 \mathrm{~A}-\mathrm{C} 16-\mathrm{H} 16 \mathrm{~B}$ & 109.5 \\
\hline $\mathrm{C} 3-\mathrm{C} 4-\mathrm{C} 5$ & $118.86(18)$ & $\mathrm{C} 13-\mathrm{C} 16-\mathrm{H} 16 \mathrm{C}$ & 109.5 \\
\hline $\mathrm{C} 3-\mathrm{C} 4-\mathrm{C} 7$ & $119.5(2)$ & $\mathrm{H} 16 \mathrm{~A}-\mathrm{C} 16-\mathrm{H} 16 \mathrm{C}$ & 109.5 \\
\hline $\mathrm{C} 5-\mathrm{C} 4-\mathrm{C} 7$ & $121.6(2)$ & $\mathrm{H} 16 \mathrm{~B}-\mathrm{C} 16-\mathrm{H} 16 \mathrm{C}$ & 109.5 \\
\hline $\mathrm{Zn} 1-\mathrm{N} 1-\mathrm{C} 1-\mathrm{C} 6$ & $-152.85(15)$ & $\mathrm{C} 4-\mathrm{C} 5-\mathrm{C} 6-\mathrm{C} 1$ & $1.3(3)$ \\
\hline $\mathrm{Zn} 1-\mathrm{N} 1-\mathrm{C} 1-\mathrm{C} 2$ & $24.00(19)$ & $\mathrm{C} 8-\mathrm{C} 5-\mathrm{C} 6-\mathrm{C} 1$ & $-177.21(19)$ \\
\hline $\mathrm{C} 6-\mathrm{C} 1-\mathrm{C} 2-\mathrm{C} 3$ & $-2.4(3)$ & $\mathrm{C} 14-\mathrm{C} 9-\mathrm{C} 10-\mathrm{C} 11$ & $-0.4(3)$ \\
\hline $\mathrm{N} 1-\mathrm{C} 1-\mathrm{C} 2-\mathrm{C} 3$ & $-179.38(16)$ & $\mathrm{N} 3-\mathrm{C} 9-\mathrm{C} 10-\mathrm{C} 11$ & $173.98(18)$ \\
\hline $\mathrm{C} 6-\mathrm{C} 1-\mathrm{C} 2-\mathrm{N} 2$ & $174.28(17)$ & $\mathrm{C} 14-\mathrm{C} 9-\mathrm{C} 10-\mathrm{N} 4$ & $-176.29(18)$ \\
\hline $\mathrm{N} 1-\mathrm{C} 1-\mathrm{C} 2-\mathrm{N} 2$ & $-2.7(2)$ & $\mathrm{N} 3-\mathrm{C} 9-\mathrm{C} 10-\mathrm{N} 4$ & $-1.9(3)$ \\
\hline $\mathrm{Zn} 1-\mathrm{N} 2-\mathrm{C} 2-\mathrm{C} 3$ & $156.68(15)$ & $\mathrm{C} 9-\mathrm{C} 10-\mathrm{C} 11-\mathrm{C} 12$ & $-0.7(3)$ \\
\hline $\mathrm{Zn} 1-\mathrm{N} 2-\mathrm{C} 2-\mathrm{C} 1$ & $-19.9(2)$ & $\mathrm{N} 4-\mathrm{C} 10-\mathrm{C} 11-\mathrm{C} 12$ & $175.16(19)$ \\
\hline $\mathrm{C} 1-\mathrm{C} 2-\mathrm{C} 3-\mathrm{C} 4$ & $1.8(3)$ & $\mathrm{C} 10-\mathrm{C} 11-\mathrm{C} 12-\mathrm{C} 13$ & $1.4(3)$ \\
\hline $\mathrm{N} 2-\mathrm{C} 2-\mathrm{C} 3-\mathrm{C} 4$ & $-174.74(17)$ & $\mathrm{C} 10-\mathrm{C} 11-\mathrm{C} 12-\mathrm{C} 15$ & $-178.3(2)$ \\
\hline $\mathrm{C} 2-\mathrm{C} 3-\mathrm{C} 4-\mathrm{C} 5$ & $0.4(3)$ & $\mathrm{C} 11-\mathrm{C} 12-\mathrm{C} 13-\mathrm{C} 14$ & $-1.1(3)$ \\
\hline $\mathrm{C} 2-\mathrm{C} 3-\mathrm{C} 4-\mathrm{C} 7$ & $-179.85(18)$ & $\mathrm{C} 15-\mathrm{C} 12-\mathrm{C} 13-\mathrm{C} 14$ & $178.7(2)$ \\
\hline $\mathrm{C} 3-\mathrm{C} 4-\mathrm{C} 5-\mathrm{C} 6$ & $-1.9(3)$ & $\mathrm{C} 11-\mathrm{C} 12-\mathrm{C} 13-\mathrm{C} 16$ & $179.2(2)$ \\
\hline $\mathrm{C} 7-\mathrm{C} 4-\mathrm{C} 5-\mathrm{C} 6$ & $178.34(19)$ & $\mathrm{C} 15-\mathrm{C} 12-\mathrm{C} 13-\mathrm{C} 16$ & $-1.0(3)$ \\
\hline $\mathrm{C} 3-\mathrm{C} 4-\mathrm{C} 5-\mathrm{C} 8$ & $176.51(19)$ & $\mathrm{C} 10-\mathrm{C} 9-\mathrm{C} 14-\mathrm{C} 13$ & $0.7(3)$ \\
\hline $\mathrm{C} 7-\mathrm{C} 4-\mathrm{C} 5-\mathrm{C} 8$ & $-3.2(3)$ & $\mathrm{N} 3-\mathrm{C} 9-\mathrm{C} 14-\mathrm{C} 13$ & $-173.60(19)$ \\
\hline
\end{tabular}




$\begin{array}{llll}\mathrm{C} 2-\mathrm{C} 1-\mathrm{C} 6-\mathrm{C} 5 & 0.9(3) & \mathrm{C} 12-\mathrm{C} 13-\mathrm{C} 14-\mathrm{C} 9 & 0.0(3) \\ \mathrm{N} 1-\mathrm{C} 1-\mathrm{C} 6-\mathrm{C} 5 & 177.72(17) & \mathrm{C} 16-\mathrm{C} 13-\mathrm{C} 14-\mathrm{C} 9 & 179.7(2)\end{array}$

Symmetry code: (i) $-x+1,-y+1,-z+1$.

Hydrogen-bond geometry $\left(A,{ }^{\circ}\right)$

\begin{tabular}{lllll}
\hline$D-\mathrm{H} \cdots A$ & $D-\mathrm{H}$ & $\mathrm{H} \cdots A$ & $D \cdots A$ & $D-\mathrm{H} \cdots A$ \\
\hline $\mathrm{O} 1-\mathrm{H} 1 W A \cdots \mathrm{N} 4^{\mathrm{ii}}$ & $0.81(3)$ & $2.13(3)$ & $2.924(3)$ & $164(2)$ \\
$\mathrm{O} 1-\mathrm{H} 1 W B \cdots \mathrm{C} 11^{\mathrm{iii}}$ & $0.80(3)$ & $2.31(3)$ & $3.1083(17)$ & $173(2)$ \\
$\mathrm{N} 1-\mathrm{H} 1 A \cdots \mathrm{C} 11^{\mathrm{iii}}$ & $0.84(2)$ & $2.55(2)$ & $3.3551(18)$ & $160.3(18)$ \\
$\mathrm{N} 1-\mathrm{H} 1 B \cdots \mathrm{N} 3^{\text {iv }}$ & $0.85(2)$ & $2.31(2)$ & $3.137(3)$ & $162.6(18)$ \\
$\mathrm{N} 2-\mathrm{H} 2 A \cdots \mathrm{C} 11^{\mathrm{v}}$ & $0.87(2)$ & $2.63(2)$ & $3.4401(19)$ & $155.3(18)$ \\
$\mathrm{N} 2-\mathrm{H} 2 B \cdots \mathrm{C} 1^{\mathrm{i}}$ & $0.81(2)$ & $2.57(2)$ & $3.3105(18)$ & $154(2)$ \\
$\mathrm{N} 3-\mathrm{H} 3 A \cdots \mathrm{C} 11^{\mathrm{v}}$ & $0.84(3)$ & $2.68(3)$ & $3.516(2)$ & $174(2)$ \\
$\mathrm{N} 3-\mathrm{H} 3 B \cdots \mathrm{Cl} 1^{\mathrm{vi}}$ & $0.87(3)$ & $2.89(2)$ & $3.3284(19)$ & $112.9(18)$ \\
$\mathrm{N} 4-\mathrm{H} 4 B \cdots \mathrm{C} 1^{\mathrm{v}}$ & $0.87(3)$ & $2.50(3)$ & $3.355(2)$ & $171(2)$
\end{tabular}

Symmetry codes: (i) $-x+1,-y+1,-z+1$; (ii) $x,-y+3 / 2, z-1 / 2$; (iii) $x, y, z-1$; (iv) $-x+1, y-1 / 2,-z+3 / 2$; (v) $-x+1, y+1 / 2,-z+3 / 2$; (vi) $-x+1,-y+1,-z+2$.

(Ilb) (Benzene-1,2-diamine- $\left.\kappa^{2} N, N^{\prime}\right)$ dichloroidozinc

Crystal data

$\left[\mathrm{ZnCl}_{2}\left(\mathrm{C}_{6} \mathrm{H}_{8} \mathrm{~N}_{2}\right)\right]$

$M_{r}=244.41$

Orthorhombic, Pnma

$a=8.4152(12) \AA$

$b=7.5141(9) \AA$

$c=14.199(2) \AA$

$V=897.8(2) \AA^{3}$

$Z=4$

$F(000)=488$

\section{Data collection}

Bruker SMART X2S benchtop diffractometer

Radiation source: XOS X-beam microfocus source

Doubly curved silicon crystal monochromator Detector resolution: 8.3330 pixels $\mathrm{mm}^{-1}$

$\omega$ scans

Absorption correction: multi-scan

(SADABS; Bruker, 2013)

\section{Refinement}

Refinement on $F^{2}$

Least-squares matrix: full

$R\left[F^{2}>2 \sigma\left(F^{2}\right)\right]=0.027$

$w R\left(F^{2}\right)=0.074$

$S=1.16$

1090 reflections

72 parameters

0 restraints

Primary atom site location: structure-invariant direct methods
$D_{\mathrm{x}}=1.808 \mathrm{Mg} \mathrm{m}^{-3}$

Mo $K \alpha$ radiation, $\lambda=0.71073 \AA$

Cell parameters from 2561 reflections

$\theta=2.8-29.2^{\circ}$

$\mu=3.27 \mathrm{~mm}^{-1}$

$T=200 \mathrm{~K}$

Prism, clear colourless

$0.60 \times 0.30 \times 0.20 \mathrm{~mm}$

$T_{\min }=0.40, T_{\max }=0.56$

4392 measured reflections

1090 independent reflections

992 reflections with $I>2 \sigma(I)$

$R_{\text {int }}=0.040$

$\theta_{\max }=27.5^{\circ}, \theta_{\min }=2.8^{\circ}$

$h=-10 \rightarrow 5$

$k=-8 \rightarrow 9$

$l=-15 \rightarrow 18$

Secondary atom site location: difference Fourier map

Hydrogen site location: mixed

$\mathrm{H}$ atoms treated by a mixture of independent and constrained refinement

$w=1 /\left[\sigma^{2}\left(F_{\mathrm{o}}^{2}\right)+(0.0377 P)^{2}\right]$

where $P=\left(F_{\mathrm{o}}^{2}+2 F_{\mathrm{c}}^{2}\right) / 3$

$(\Delta / \sigma)_{\max }<0.001$

$\Delta \rho_{\max }=0.41$ e $\AA^{-3}$

$\Delta \rho_{\min }=-0.58$ e $\AA^{-3}$ 


\section{Special details}

Geometry. All esds (except the esd in the dihedral angle between two 1.s. planes) are estimated using the full covariance matrix. The cell esds are taken into account individually in the estimation of esds in distances, angles and torsion angles; correlations between esds in cell parameters are only used when they are defined by crystal symmetry. An approximate (isotropic) treatment of cell esds is used for estimating esds involving l.s. planes.

Refinement. Refined as a 2-component inversion twin.

Fractional atomic coordinates and isotropic or equivalent isotropic displacement parameters $\left(\AA^{2}\right)$

\begin{tabular}{lllll}
\hline & $x$ & $y$ & $z$ & $U_{\text {iso }} * U_{\text {eq }}$ \\
\hline Zn1 & $0.12938(3)$ & 0.75 & $0.38034(2)$ & $0.03201(15)$ \\
$\mathrm{N} 1$ & $0.3669(2)$ & 0.75 & $0.3494(2)$ & $0.0343(6)$ \\
$\mathrm{H} 1$ & $0.388(2)$ & $0.661(3)$ & $0.318(2)$ & $0.040(7)^{*}$ \\
$\mathrm{~N} 2$ & $0.2002(3)$ & 0.75 & $0.51812(18)$ & $0.0354(6)$ \\
$\mathrm{H} 2$ & $0.165(3)$ & $0.666(3)$ & $0.550(2)$ & $0.047(7)^{*}$ \\
$\mathrm{C} 11$ & $0.00184(5)$ & $0.50585(6)$ & $0.33279(4)$ & $0.04089(18)$ \\
$\mathrm{C} 1$ & $0.4559(3)$ & 0.75 & $0.4370(2)$ & $0.0294(6)$ \\
$\mathrm{C} 2$ & $0.3728(3)$ & 0.75 & $0.5215(2)$ & $0.0284(6)$ \\
$\mathrm{C} 3$ & $0.4543(4)$ & 0.75 & $0.6066(2)$ & $0.0402(7)$ \\
$\mathrm{H} 3$ & 0.3973 & 0.75 & 0.6644 & $0.048^{*}$ \\
$\mathrm{C} 4$ & $0.6198(4)$ & 0.75 & $0.6068(3)$ & $0.0482(9)$ \\
$\mathrm{H} 4$ & 0.6761 & 0.75 & 0.6648 & $0.058^{*}$ \\
$\mathrm{C} 5$ & $0.7015(4)$ & 0.75 & $0.5231(3)$ & $0.0493(8)$ \\
H5 & 0.8144 & 0.75 & 0.5237 & $0.059^{*}$ \\
C6 & $0.6220(3)$ & 0.75 & $0.4382(3)$ & $0.0423(8)$ \\
H6 & 0.6798 & 0.75 & 0.3807 & $0.051^{*}$ \\
& & & &
\end{tabular}

Atomic displacement parameters $\left(\AA^{2}\right)$

\begin{tabular}{lllllll}
\hline & $U^{11}$ & $U^{22}$ & $U^{33}$ & $U^{12}$ & $U^{13}$ & $U^{23}$ \\
\hline $\mathrm{Zn} 1$ & $0.0301(2)$ & $0.0408(3)$ & $0.0251(2)$ & 0 & $-0.00244(10)$ & 0 \\
$\mathrm{~N} 1$ & $0.0345(12)$ & $0.0468(18)$ & $0.0216(12)$ & 0 & $0.0024(8)$ & 0 \\
$\mathrm{~N} 2$ & $0.0346(12)$ & $0.0469(17)$ & $0.0246(12)$ & 0 & $0.0041(9)$ & 0 \\
$\mathrm{C} 11$ & $0.0502(3)$ & $0.0367(3)$ & $0.0358(3)$ & $-0.0066(2)$ & $-0.0058(2)$ & $0.0032(2)$ \\
$\mathrm{C} 1$ & $0.0341(12)$ & $0.0282(14)$ & $0.0259(13)$ & 0 & $-0.0001(10)$ & 0 \\
$\mathrm{C} 2$ & $0.0309(13)$ & $0.0289(14)$ & $0.0253(14)$ & 0 & $-0.0005(9)$ & 0 \\
$\mathrm{C} 3$ & $0.0498(16)$ & $0.0423(17)$ & $0.0283(15)$ & 0 & $-0.0035(12)$ & 0 \\
$\mathrm{C} 4$ & $0.0487(18)$ & $0.051(2)$ & $0.045(2)$ & 0 & $-0.0201(14)$ & 0 \\
$\mathrm{C} 5$ & $0.0331(14)$ & $0.053(2)$ & $0.061(2)$ & 0 & $-0.0120(14)$ & 0 \\
$\mathrm{C} 6$ & $0.0331(14)$ & $0.051(2)$ & $0.0425(19)$ & 0 & $0.0048(11)$ & 0 \\
\hline
\end{tabular}

Geometric parameters $\left(\AA,{ }^{\circ}\right)$

\begin{tabular}{llll}
\hline $\mathrm{Zn} 1-\mathrm{C} 11$ & $2.2301(5)$ & $\mathrm{C} 5-\mathrm{C} 6$ & $1.379(5)$ \\
$\mathrm{Zn} 1-\mathrm{C} 11^{\mathrm{i}}$ & $2.2301(5)$ & $\mathrm{C} 5-\mathrm{H} 5$ & 0.95 \\
$\mathrm{Zn} 1-\mathrm{N} 1$ & $2.047(2)$ & $\mathrm{C} 4-\mathrm{C} 5$ & $1.373(5)$ \\
$\mathrm{Zn} 1-\mathrm{N} 2$ & $2.045(3)$ & $\mathrm{C} 4-\mathrm{H} 4$ & 0.95 \\
$\mathrm{~N} 2-\mathrm{C} 2$ & $1.453(3)$ & $\mathrm{C} 3-\mathrm{C} 4$ & $1.393(4)$
\end{tabular}




\begin{tabular}{|c|c|c|c|}
\hline $\mathrm{N} 2-\mathrm{H} 2$ & $0.83(3)$ & $\mathrm{C} 3-\mathrm{H} 3$ & 0.95 \\
\hline $\mathrm{N} 1-\mathrm{C} 1$ & $1.452(4)$ & $\mathrm{C} 2-\mathrm{C} 3$ & $1.389(4)$ \\
\hline $\mathrm{N} 1-\mathrm{H} 1$ & $0.83(3)$ & $\mathrm{C} 1-\mathrm{C} 2$ & $1.388(4)$ \\
\hline $\mathrm{C} 6-\mathrm{H} 6$ & 0.95 & $\mathrm{C} 1-\mathrm{C} 6$ & $1.398(3)$ \\
\hline $\mathrm{C} 11-\mathrm{Zn} 1-\mathrm{C} 11^{\mathrm{i}}$ & $110.70(3)$ & $\mathrm{C} 4-\mathrm{C} 5-\mathrm{C} 6$ & $120.9(3)$ \\
\hline $\mathrm{N} 1-\mathrm{Zn} 1-\mathrm{N} 2$ & $85.45(10)$ & $\mathrm{C} 6-\mathrm{C} 5-\mathrm{H} 5$ & 119.5 \\
\hline $\mathrm{N} 1-\mathrm{Zn} 1-\mathrm{Cl} 1$ & $113.89(4)$ & $\mathrm{C} 4-\mathrm{C} 5-\mathrm{H} 5$ & 119.5 \\
\hline $\mathrm{N} 2-\mathrm{Zn} 1-\mathrm{Cl1}{ }^{\mathrm{i}}$ & $115.46(3)$ & $\mathrm{C} 5-\mathrm{C} 4-\mathrm{C} 3$ & $119.9(3)$ \\
\hline $\mathrm{N} 2-\mathrm{Zn} 1-\mathrm{Cl} 1$ & $115.46(3)$ & $\mathrm{C} 5-\mathrm{C} 4-\mathrm{H} 4$ & 120.0 \\
\hline $\mathrm{N} 1-\mathrm{Zn} 1-\mathrm{C} 11^{\mathrm{i}}$ & $113.89(4)$ & $\mathrm{C} 3-\mathrm{C} 4-\mathrm{H} 4$ & 120.0 \\
\hline $\mathrm{Zn} 1-\mathrm{N} 2-\mathrm{H} 2$ & $114.1(19)$ & $\mathrm{C} 2-\mathrm{C} 3-\mathrm{C} 4$ & $119.7(3)$ \\
\hline $\mathrm{C} 2-\mathrm{N} 2-\mathrm{H} 2$ & $110.0(17)$ & $\mathrm{C} 4-\mathrm{C} 3-\mathrm{H} 3$ & 120.2 \\
\hline $\mathrm{C} 2-\mathrm{N} 2-\mathrm{Zn} 1$ & $108.84(19)$ & $\mathrm{C} 2-\mathrm{C} 3-\mathrm{H} 3$ & 120.2 \\
\hline $\mathrm{Zn} 1-\mathrm{N} 1-\mathrm{H} 1$ & $109.0(14)$ & $\mathrm{C} 1-\mathrm{C} 2-\mathrm{C} 3$ & $120.2(2)$ \\
\hline $\mathrm{C} 1-\mathrm{N} 1-\mathrm{H} 1$ & $111.0(18)$ & $\mathrm{C} 3-\mathrm{C} 2-\mathrm{N} 2$ & $121.5(3)$ \\
\hline $\mathrm{C} 1-\mathrm{N} 1-\mathrm{Zn} 1$ & $108.62(18)$ & $\mathrm{C} 1-\mathrm{C} 2-\mathrm{N} 2$ & $118.4(2)$ \\
\hline $\mathrm{C} 5-\mathrm{C} 6-\mathrm{C} 1$ & $119.7(3)$ & $\mathrm{C} 2-\mathrm{C} 1-\mathrm{C} 6$ & $119.6(3)$ \\
\hline $\mathrm{C} 5-\mathrm{C} 6-\mathrm{H} 6$ & 120.2 & $\mathrm{C} 6-\mathrm{C} 1-\mathrm{N} 1$ & $121.7(3)$ \\
\hline $\mathrm{C} 1-\mathrm{C} 6-\mathrm{H} 6$ & 120.2 & $\mathrm{C} 2-\mathrm{C} 1-\mathrm{N} 1$ & $118.7(2)$ \\
\hline $\mathrm{Zn} 1-\mathrm{N} 1-\mathrm{C} 1-\mathrm{C} 2$ & 0 & $\mathrm{C} 1-\mathrm{C} 2-\mathrm{C} 3-\mathrm{C} 4$ & $0.0000(10)$ \\
\hline $\mathrm{Zn} 1-\mathrm{N} 1-\mathrm{C} 1-\mathrm{C} 6$ & 180.0 & $\mathrm{~N} 2-\mathrm{C} 2-\mathrm{C} 3-\mathrm{C} 4$ & $180.0000(10)$ \\
\hline $\mathrm{C} 6-\mathrm{C} 1-\mathrm{C} 2-\mathrm{C} 3$ & $0.0000(10)$ & $\mathrm{C} 2-\mathrm{C} 3-\mathrm{C} 4-\mathrm{C} 5$ & $0.0000(10)$ \\
\hline $\mathrm{N} 1-\mathrm{C} 1-\mathrm{C} 2-\mathrm{C} 3$ & $180.0000(10)$ & $\mathrm{C} 3-\mathrm{C} 4-\mathrm{C} 5-\mathrm{C} 6$ & $0.0000(10)$ \\
\hline $\mathrm{C} 6-\mathrm{C} 1-\mathrm{C} 2-\mathrm{N} 2$ & 180.0 & $\mathrm{C} 4-\mathrm{C} 5-\mathrm{C} 6-\mathrm{C} 1$ & $0.0000(10)$ \\
\hline $\mathrm{N} 1-\mathrm{C} 1-\mathrm{C} 2-\mathrm{N} 2$ & $0.0000(10)$ & $\mathrm{C} 2-\mathrm{C} 1-\mathrm{C} 6-\mathrm{C} 5$ & $0.0000(10)$ \\
\hline $\mathrm{Zn} 1-\mathrm{N} 2-\mathrm{C} 2-\mathrm{C} 1$ & $0.0000(10)$ & $\mathrm{N} 1-\mathrm{C} 1-\mathrm{C} 6-\mathrm{C} 5$ & $180.0000(10)$ \\
\hline $\mathrm{Zn} 1-\mathrm{N} 2-\mathrm{C} 2-\mathrm{C} 3$ & $180.0000(10)$ & & \\
\hline
\end{tabular}

Symmetry code: (i) $x,-y+3 / 2, z$.

Hydrogen-bond geometry $\left(\AA,{ }^{\circ}\right)$

\begin{tabular}{lllll}
\hline$D-\mathrm{H} \cdots A$ & $D-\mathrm{H}$ & $\mathrm{H} \cdots A$ & $D \cdots A$ & $D-\mathrm{H} \cdots A$ \\
\hline $\mathrm{N} 1-\mathrm{H} 1 \cdots \mathrm{Cl} 1^{\mathrm{ii}}$ & $0.83(3)$ & $2.61(3)$ & $3.368(2)$ & $152(2)$ \\
$\mathrm{N} 2-\mathrm{H} 2 \cdots \mathrm{Cl} 1^{\mathrm{iii}}$ & $0.83(3)$ & $2.53(3)$ & $3.327(2)$ & $160(2)$ \\
\hline
\end{tabular}

Symmetry codes: (ii) $x+1 / 2, y,-z+1 / 2$; (iii) $-x,-y+1,-z+1$. 